\title{
HEDGEHOG/GLI-E2F1 axis modulates iASPP expression and function and regulates melanoma cell growth
}

\author{
S Pandolfi ${ }^{1,4}$, V Montagnani ${ }^{1,4}, A$ Lapucci ${ }^{2}$ and B Stecca ${ }^{*, 1,3}$
}

HEDGEHOG $(\mathrm{HH})$ signaling is a key regulator of tissue development and its aberrant activation is involved in several cancer types, including melanoma. We and others have shown a reciprocal cross talk between $\mathrm{HH}$ signaling and p53, whose function is often impaired in melanoma. Here we present evidence that both GLI1 and GLI2, the final effectors of HH signaling, regulate the transcription factor E2F1 in melanoma cells, by binding to a functional non-canonical GLI consensus sequence. Consistently, we find a significant correlation between E2F1 and PATCHED1 (PTCH1), GLI1 and GLI2 expression in human melanomas. Functionally, we find that E2F1 is a crucial mediator of $\mathrm{HH}$ signaling and it is required for melanoma cell proliferation and xenograft growth induced by activation of the HH pathway. Interestingly, we present evidence that the HH/GLI-E2F1 axis positively modulates the inhibitor of apoptosis-stimulating protein of p53 (iASPP) at multiple levels. HH activation induces iASPP expression through E2F1, which directly binds to iASPP promoter. HH pathway also contributes to iASPP function, by the induction of Cyclin B1 and by the E2F1-dependent regulation of CDK1, which are both involved in iASPP activation. Our data show that activation of HH signaling enhances proliferation in presence of E2F1 and promotes apoptosis in its absence or upon CDK1 inhibition, suggesting that E2F1/ iASPP dictates the outcome of HH signaling in melanoma. Together, these findings identify a novel HH/GLI-E2F1-iASPP axis that regulates melanoma cell growth and survival, providing an additional mechanism through which $\mathrm{HH}$ signaling restrains p53 proapoptotic function.

Cell Death and Differentiation (2015) 22, 2006-2019; doi:10.1038/cdd.2015.56; published online 29 May 2015

Hedgehog $(\mathrm{HH})$ signaling is a conserved pathway that directs embryonic patterning through the temporal and spatial regulation of cellular proliferation and differentiation. ${ }^{1,2}$ During development, the loss of $\mathrm{HH}$ signaling results in severe abnormalities in mice and humans. ${ }^{3-5}$ In the adult it is mostly active in stem/progenitor cells, where it regulates tissue homeostasis, repair and regeneration. ${ }^{6}$ Conversely, unrestrained $\mathrm{HH}$ pathway activation is implicated in a variety of tumors, including those of the skin. ${ }^{7,8}$ Secreted $\mathrm{HH}$ ligands trigger downstream signaling by binding to the transmembrane receptor Patched (PTCH1). PTCH1 relieves its inhibition on the G protein-coupled receptor Smoothened (SMO), which triggers an intracellular signaling cascade regulating the formation of the zinc finger transcription factors GLI2 and GLI3 and their translocation into the nucleus. ${ }^{9,10}$ Both GLI1 and GLI2 act as main mediators of $\mathrm{HH}$ signaling in cancer by directly controlling the transcription of target genes, several of which are involved in proliferation. ${ }^{11,12}$

Cutaneous melanoma arises from malignant transformation of melanocytes and is the most aggressive form of skin cancer, with poor prognosis in late stages. ${ }^{13}$ In contrast to other tumors, $>80 \%$ of melanomas retain wild-type (wt) p53. ${ }^{14,15}$
Nevertheless, p53 tumor-suppressor activity is impaired by various mechanisms, including the deletion of the CDKN2A locus ${ }^{16,17}$ or MDM2 and MDMX overexpression. ${ }^{18-21}$ Recently, the inhibitor of apoptosis-stimulating protein of p53 (iASPP), ${ }^{22,23}$ which is frequently upregulated in human cancers, ${ }^{24-29}$ has been proposed to hamper p53 function in melanoma. ${ }^{21}$

$\mathrm{HH}$ pathway is often activated in human melanoma, where it is required for proliferation and survival both in vitro and in vivo ${ }^{30-32}$ and it drives self-renewal and tumorigenicity of melanoma cancer stem cells (CSCs). ${ }^{33}$ Previous data indicate a reciprocal cross talk between $\mathrm{HH}$ signaling and p53. Activation of the $\mathrm{HH}$ pathway impairs p53 function by increasing MDM2 levels. ${ }^{34}$ In turn, p53 inhibits GLI1 levels, nuclear localization and transcriptional activity ${ }^{35}$ and, upon DNA damage, promotes GLI1 degradation through PCAF induction. $^{36}$

Restoration of wt p53 function is an attractive therapeutic approach for melanoma, and recent evidence support E2F1 as a biomarker to predict the outcome of the treatment with inhibitors of MDM2/p53 interaction. ${ }^{37}$ E2F1 is a key transcriptional regulator of proliferation ${ }^{38-40}$ whose aberrant activation

\footnotetext{
${ }^{1}$ Laboratory of Tumor Cell Biology, Core Research Laboratory-Istituto Toscano Tumori (CRL-ITT), Florence, Italy; ${ }^{2}$ Department of Health Sciences, Section of Clinical Pharmacology and Oncology, University of Florence, Florence, Italy and ${ }^{3}$ Department of Oncology, Azienda Ospedaliero-Universitaria Careggi, Florence, Italy

${ }^{*}$ Corresponding author: B Stecca, Laboratory of Tumor Cell Biology, Core Research Laboratory-Istituto Toscano Tumori (CRL-ITT), Viale Pieraccini 6, Florence 50139 Italy; Tel: +39055 7944567; Fax: +39 055 7944586. E-mail: barbara.stecca@ittumori.it

${ }^{4}$ These authors contributed equally to this work.

Abbreviations: HH, Hedgehog; PTCH1, Patched1; SMO, Smoothened; iASPP, inhibitor of apoptosis-stimulating protein of p53; CDK1, cyclin-dependent kinase 1; PIG3, p53-inducible gene 3; p53AIP1, p53-regulated apoptosis-inducing protein 1; BCL2, B-cell lymphoma 2; BCL-XL, B-cell lymphoma extra-large; CSC, cancer stem cells; GLIBS, GLI-binding site; shRNA, short hairpin RNA; EMSA, electrophoretic mobility shift assay; JNJ, JNJ-7706621.

Received 08.10.14; revised 26.3.15; accepted 08.4.15; Edited by B Zhivotovsky; published online 29.5.15
} 
has been described in various tumor types. ${ }^{41,42}$ In melanoma E2F1 drives metastasis formation by regulating the epidermal growth factor receptor. ${ }^{43}$ Notably, E2F1 has been shown to be a $\mathrm{HH}$-responsive gene in cerebellar granular neural precursors, medulloblastoma and keratinocytes. ${ }^{44-46}$ However, it is not known which effects induced by $\mathrm{HH}$ signaling activation in melanoma are mediated by E2F1. Here we demonstrate that both GLI1 and GLI2 induce E2F1 expression in melanoma by binding to the E2F1 promoter. Importantly, we show that E2F1 dictates the outcome of $\mathrm{HH}$ pathway activation by controlling the expression and function of iASPP.

\section{Results}

HH signaling modulates E2F1 expression in melanoma cells. To investigate whether $\mathrm{HH}$ pathway modulates E2F1 expression in melanoma, we inhibited $\mathrm{HH}$ signaling by $\mathrm{SMO}$ silencing, transducing patient-derived SSM2c and M26c, and commercial A375 melanoma cells with a replicationincompetent lentivirus expressing a short interference RNA targeting SMO (LV-shSMO). ${ }^{33}$ Quantitative real-time PCR (qPCR) analysis showed strong reduction of mRNA levels of SMO and of the two $\mathrm{HH}$ targets GLI1 and PTCH1, confirming the inhibition of $\mathrm{HH}$ signaling (Figure 1a). Notably, SMO silencing decreased E2F1 mRNA and protein levels compared with the control (Figures $1 \mathrm{a}$ and $\mathrm{b}$ and Supplementary Figure 1a) in all three melanoma cells. In addition, GLI2 silencing (LV-shGLI2) reduced E2F1 mRNA levels in A375 cells, which express high levels of GLI2 (Supplementary Figures $1 \mathrm{~b}$ and $\mathrm{c}$ and Supplementary Figure 2a). Conversely, activation of the $\mathrm{HH}$ pathway by silencing the negative regulator PTCH1 (LV-shPTCH1; ref. 35) increased GLI1 and E2F1 mRNA levels (Figure 1c). Transfection of Myc-tagged GLI1 or GLI2 increased the endogenous E2F1 protein in SSM2c and M26c cells (Figures 1d and e). Altogether these results suggest that E2F1 expression in melanoma cells is affected by the modulation of the $\mathrm{HH}$ signaling. A publicly available microarray data set in 31 primary and 73 metastatic melanomas (GEO-46517; ref. 47) was analyzed. In support of the relevance of modulation of E2F1 by the $\mathrm{HH}$ pathway, a significant correlation between E2F1 and PTCH1, GLI1 and GLI2 expression was found in metastatic melanomas, whereas in primary melanomas E2F1 correlated only with GLI2 (Figure 1f), suggesting an association between $\mathrm{HH}$ pathway activation and E2F1 expression. As a further confirm of this modulation, a significant correlation between E2F1 and GLI1 mRNA (Supplementary Figures $2 b$ and $c$ ) was found in a panel of eight patient-derived melanoma cells and six melanoma cell lines (Supplementary Table 1).

GLI1 and GLI2 bind to a non-canonical GLI consensus sequence at E2F1 promoter. To test whether E2F1 expression was directly regulated by GLI1 or GLI2, we performed chromatin immunoprecipitation (ChIP) assay in M26c, SSM2c and A375 cells in which $\mathrm{HH}$ pathway was activated by $\mathrm{PTCH} 1$ silencing. qPCR analysis on the immunoprecipitated DNA showed that both endogenous GLI1 and GLI2 bound to E2F1 promoter (Figure 2a and Supplementary Figure 3). The activation of $\mathrm{HH}$ pathway resulted in increased GLI1 binding to $\mathrm{PTCH} 1$ promoter (used as positive control) in M26c and SSM2c cells, and increased GLI2 binding in A375 cells. The binding of GLI1 and GLI2 to E2F1 promoter was not further increased by the $\mathrm{HH}$ pathway activation, and it was stronger than that to $\mathrm{PTCH} 1$ promoter. Nevertheless, ChIP experiment showed that the activation of $\mathrm{HH}$ signaling increased E2F1 binding to its promoter in the proximity of a conserved E2F-binding site (Supplementary Figure 4). This suggests that the activation of the $\mathrm{HH}$ pathway increases E2F1 levels, which could then contribute to sustain the transactivation of its own promoter, as already shown. ${ }^{48}$

Bioinformatic analysis did not identify any canonical GLI consensus sequence (GACCACCCA) ${ }^{49}$ in the E2F1 promoter. However, GLI transcription factors are able to bind to variant GLI-binding sites (GLI-BSs) with relatively low affinity, still leading to strong transcriptional activation ${ }^{50,51}$ (Figure $2 b$ ). We thus speculated that the E2F1 promoter might contain a degenerate GLI-BS. To identify the region responsible for the modulation by GLI transcription factors, we cloned three different fragments of the E2F1 promoter upstream of the luciferase gene $(-132,-269$ and $-656 \mathrm{bp}$; Figure 2c). We transfected SSM2c and M26c cells with the reporter vector along with GLI1, GLI2 or E2F1, the latter used as positive control because it binds to the E2F1 promoter close to the transcription start site. ${ }^{48}$ The assay showed that both GLI1 and GLI2 transactivated the reporter driven by the -269 and -656 bp fragments, but not by the -132 bp fragment (Figure 2c). As expected, E2F1 transactivated all the three regions (Figure $2 \mathrm{c}$ ). These results indicate the presence of a functional GLI-BS between - 132 and - 269 bp upstream the E2F1 transcription start site.

To precisely map the GLI-BS, we identified two putative degenerated GLI consensus sequences (Figure 2d; site1 and site2) and we mutated each of them in two positions crucial for the efficiency of GLI-binding. ${ }^{50}$ Reporter assay showed that the disruption of the site1 (Mut1) prevented the transactivation of the reporter by GLI1 and GLI2 in both SSM2c and M26c cells, whereas the mutation of the site2 (Mut2) did not have any effect (Figure 2e). Electrophoretic mobility shift assay (EMSA) in $\mathrm{HH}$ signaling competent murine NIH3T3 cells showed the direct binding of Gli1/2 to site1. The activation of the $\mathrm{HH}$ pathway by stimulation with $\mathrm{SMO}$ agonist $\mathrm{SAG}^{52}$ led to the formation of a DNA-binding complex, which was competed with unlabeled probe. Preincubation of cell extracts with antiGLI1 or anti-GLI2 antibodies decreased the formation of this DNA complex, suggesting the presence of Gli1 and Gli2 proteins (Figure 2f). Western blot analysis confirmed the activation of the Hh signaling upon SAG treatment (Figure $2 \mathrm{~g}$ ). Altogether these results indicate that GLI1 and GLI2 directly interact with the E2F1 promoter by binding to a non-canonical GLI consensus sequence (CGCCTCCAG).

E2F1 dictates the outcome of $\mathrm{HH}$ pathway activation in melanoma cells. Our data indicate that in melanoma cells $\mathrm{HH}$ signaling directly controls the expression of E2F1, a key transcriptional regulator of proliferation. ${ }^{38-40}$ We and others have previously shown that $\mathrm{HH}$ signaling is required for melanoma growth and stemness. ${ }^{30-33}$ To investigate the effects of the $\mathrm{HH}$ pathway that are mediated by E2F1 in melanoma, we knocked down E2F1 after the activation of the 

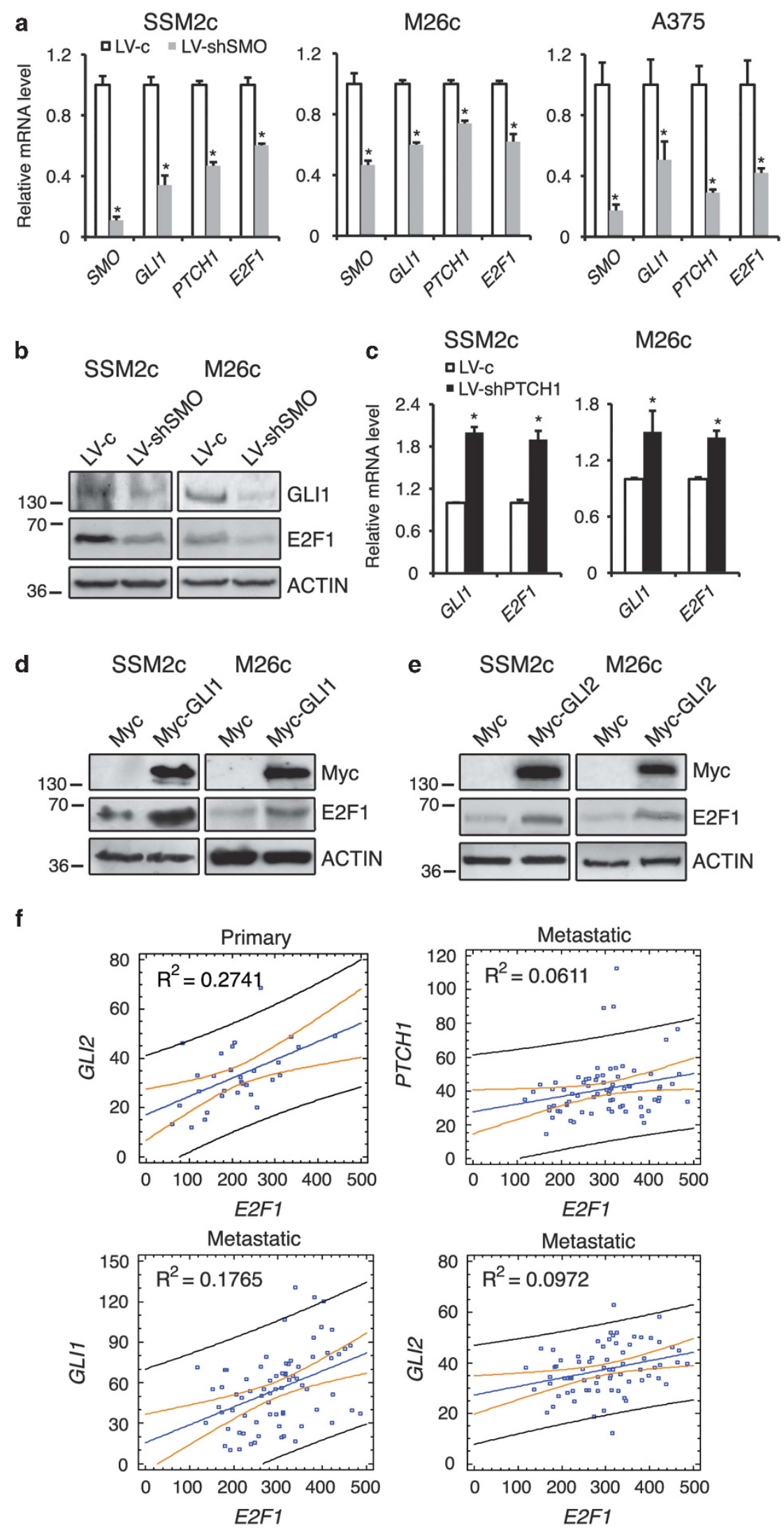
$\mathrm{HH}$ signaling. We silenced E2F1 by transducing cells with LVshE2F1-1 and LV-shE2F1-2 lentiviruses, which strongly reduced E2F1 mRNA and protein levels (Supplementary Figures $5 \mathrm{a}$ and $\mathrm{b}$ ). To investigate the presence of possible compensatory effects by other E2F members upon E2F1 silencing, we measured the expression of the activator E2F members E2F2, E2F3A and the repressor member E2F4. No significant differences were detected in E2F2, E2F3A and E2F4 expression (Supplementary Figures $5 \mathrm{c}$ and d) in two melanoma cells. To activate $\mathrm{HH}$ signaling, we silenced $\mathrm{PTCH}$, thus mimicking the canonical pathway activation. M26c, SSM2c and A375 cells were transduced with LVshPTCH1 or LV-shE2F1, alone or in combination. qPCR and western blot analysis showed an increase of GLI1, GLI2 and E2F1 expression upon $\mathrm{PTCH} 1$ silencing, confirming the activation of the $\mathrm{HH}$ pathway and a strong decrease of E2F1 upon E2F1 silencing (Figure $3 a$ and Supplementary Figure 6). To investigate the effect of E2F1 in mediating $\mathrm{HH}$-induced cell growth, cells transduced as described above were seeded and allowed to grow for 7 days. In all melanoma cell lines, LV-shPTCH1 increased the cell number compared with LV-c, whereas LV-shE2F1 reduced it. Interestingly, in cells transduced with both LV-shPTCH1 and LV-shE2F1 the reduction of cell growth was greater than in cells transduced with LV-shE2F1 alone (Figure 3b).

Cytometric analysis of proliferation index $(\mathrm{Pi})$ showed that $\mathrm{HH}$ pathway activation increased cell proliferation, as expected. E2F1 silencing by itself did not significantly affect $\mathrm{Pi}$, but it markedly decreased the effect of LV-shPTCH1 (Supplementary Figures $7 \mathrm{a}$ and $\mathrm{c}$ ). Cell cycle distribution did not vary, although LV-shPTCH1/LV-shE2F1-transduced cells presented a significant increase in the population with subG DNA content, corresponding to the apoptotic fraction (Supplementary Figures $7 b, d$ and e). Annexin V-PE/7-AAD labeling showed a consistent increase in the percentage of cells in late apoptosis when E2F1 was silenced in combination with $\mathrm{HH}$ signaling activation (Figure $3 \mathrm{c}$ ). The apoptotic response observed in LV-shPTCH1/LV-shE2F1-transduced cells was consistent with the increased expression of the proapoptotic p53-target genes PIG3 and p53AIP1, and decreased levels of the antiapoptotic factors $B C L-X L$ and BCL2 (Figures 3d and e and Supplementary Figure 8), suggesting the involvement of p53 in controlling cell death. Altogether these data indicate that E2F1 is critical to sustain melanoma cell growth induced by the activation of the $\mathrm{HH}$ signaling and suggest that E2F1 controls the balance between cell proliferation and apoptosis upon $\mathrm{HH}$ pathway activation.

HH signaling regulates iASPP expression and function through E2F1. Recent evidence indicate that melanomas harboring wt p53 express high levels of phosphorylated iASPP, which functionally inactivates $p 53 .{ }^{21}$ Little is known about the regulation of iASPP expression, therefore we sought to investigate whether it might be affected by the $\mathrm{HH}$ signaling. qPCR analysis showed that in M26c, SSM2c and A375 cells inhibition of $\mathrm{HH}$ pathway by $\mathrm{SMO}$ silencing produced a decrease of IASPP mRNA (Figure 4a). Conversely, activation of the $\mathrm{HH}$ signaling by $\mathrm{PTCH} 1$ silencing resulted in $i A S P P$ induction (Figure 4b). Two different antibodies targeting the $\mathrm{N}$ - or C-terminal regions of iASPP, both validated by iASPP silencing (Supplementary Figure 9), were used to assess the impact of $\mathrm{HH}$ pathway activation on iASPP protein. Although we were not able to clearly detect a slow-migrating iASPP that could be compatible with the Cyclin B1/CDK1-phosphorylated form, western blot analysis showed that PTCH1 depletion increased iASPP protein level, confirming $\mathrm{qPCR}$ results (Figures $4 \mathrm{c}$ and $5 \mathrm{c}$ ). Because iASPP inhibits p53 function by interacting with it, ${ }^{21}$ we assessed whether activation of the $\mathrm{HH}$ pathway might affect iASPP-p53 interaction. Indeed, in M26c and SSM2c cells transduced with LV-shPTCH1, iASPP co-immunoprecipitated p53 more efficiently than in control cells (Figure 4c), suggesting that $\mathrm{HH}$ signaling activation increases iASPPp53 binding, contributing to restrain p53 function through iASPP.

Because PTCH1 silencing, combined with E2F1 depletion, produced a marked apoptotic response with the upregulation of p53-target genes (Figures 3c, $d$ and e and Supplementary Figure 8), we speculated that E2F1 might mediate iASPP induction upon $\mathrm{HH}$ signaling activation. Interrogation of publicly available databases revealed the presence of a conserved E2F-binding site within iASPP promoter (Supplementary Figure 10a). Indeed, ChIP analysis showed that E2F1 bound to $i A S P P$ promoter and that its binding was greatly increased by $\mathrm{HH}$ pathway activation (Figure $5 \mathrm{a}$ and Supplementary Figure 10b). To assess whether E2F1 mediates iASPP induction upon $\mathrm{HH}$ signaling activation, we performed western blot and qPCR analyses in melanoma cells transduced with LV-shPTCH1 and/or LV-shE2F1. PTCH1 silencing induced iASPP levels in an E2F1-dependent manner, because E2F1 depletion abolished the effect of $\mathrm{HH}$ pathway activation (Figures 5b and $c$ and Supplementary Figure 11). Altogether these data indicate that $\mathrm{HH}$ signaling modulates iASPP expression through E2F1.

The ability of iASPP to interfere with p53 function depends mainly on its phosphorylation by CDK1/Cyclin B1 complex. ${ }^{21}$ We thus investigated whether $\mathrm{HH}$ pathway might affect also CDK1 or Cyclin B1 levels. Western blot analysis on melanoma cells transduced with LV-shPTCH1 and/or LV-shE2F1 showed that CDK1 expression was completely abolished upon E2F1 silencing (Figure 5c), consistently with a previous report

Figure $1 \mathrm{HH}$ pathway modulates the expression of E2F1 in melanoma. (a) Expression of the HH pathway components (SMO, GL11 and PTCH1) and E2F1 in SSM2c, M26c and A375 cells transduced with LV-c or LV-shSMO lentiviruses, measured by quantitative PCR (qPCR). The $y$-axis represents the expression ratio of gene/(EIF2 $\alpha+H P R T$ average). Data represent mean \pm S.E.M. of three independent experiments. ${ }^{*} P<0.05$. (b) Western blot analysis showing the decrease of endogenous GLI1 and E2F1 proteins upon SMO silencing in SSM2c and M26c cells. $\beta$-actin served as loading control. (c) Expression of GLI1 and E2F1 in SSM2c and M26c cells transduced with LV-c or LV-shPTCH1 lentiviruses, measured by qPCR. The $y$-axis represents the expression ratio of gene/(EIF2 $\alpha+H P R T$ average). Data represent mean \pm S.E.M. of three independent experiments. ${ }^{*} P<0.05$. (d,e) Western blot analysis showing an increase of endogenous E2F1 protein upon Myc-tagged GLI1 (d) or Myc-tagged GLI2 (e) overexpression in SSM2c and M26c cells. $\beta$-actin served as loading control. (f) Positive correlation between the levels of E2F1 and GLI2 in primary melanomas $\left(R^{2}=0.2741, P=0.0025\right)$, E2F1 and PTCH1 $\left(R^{2}=0.0611, P=0.0351\right), G L / 1\left(R^{2}=0.1765, P=0.0002\right)$ and $G L / 2\left(R^{2}=0.0972, P=0.0072\right)$ transcripts in metastatic melanomas 
indicating that E2F1 regulates CDK1 expression. ${ }^{53}$ ChIP analysis revealed a strong enrichment of E2F1 on the CDK1 promoter (Figure 5d and Supplementary Figure 12), supporting a direct regulation, ${ }^{54}$ and the binding was further increased upon $\mathrm{HH}$ pathway activation. PTCH1 depletion also increased Cyclin B1 levels, as previously reported ${ }^{55,56}$ and this increase was independent of E2F1, because it occurred also in cells transduced with LV-shPTCH1/LV-shE2F1 (Figure 5c). These data indicate that $\mathrm{HH}$ signaling contributes to both iASPP induction, through E2F1, and to iASPP function, via Cyclin B1 and E2F1-dependent regulation of CDK1.

$\mathrm{HH}$ signaling contributes to iASPP function through E2F1dependent regulation of CDK1 (Figure 5). Therefore we speculated that the inhibition of CDK1 activity with the CDK1 inhibitor JNJ-7706621 (JNJ, $500 \mathrm{nM}),{ }^{57}$ which was recently shown to block iASPP phosphorylation, ${ }^{21}$ would mimic the effect of E2F1 depletion. JNJ treatment markedly reduced the ability of iASPP to co-immunoprecipitate p53 (Figure 6a), confirming the efficacy of CDK1 inhibition. Growth assay showed that JNJ treatment produced a stronger reduction in the cell number of melanoma cells with activated $\mathrm{HH}$ pathway compared with the control (Figure 6b), paralleling the results obtained by the E2F1 silencing (Figure 3b). Cytometric analysis revealed that cells with activated $\mathrm{HH}$ signaling were more sensitive to CDK1 inhibition than the control cells, undergoing increased apoptosis and cell death upon JNJ treatment (Figures $6 \mathrm{c}$ and d). Altogether these data show that the inhibition of CDK1 activity produces the similar increase of apoptosis as E2F1 depletion in cells with activated $\mathrm{HH}$ pathway, suggesting that E2F1 determines the outcome of $\mathrm{HH}$ signaling activation by controlling not only iASPP expression but also its function through CDK1.

\section{Depletion of E2F1 suppresses $\mathrm{HH}-$ mediated melanoma xenograft growth. To investigate whether E2F1 regulates $\mathrm{HH}$-induced growth of melanoma xenografts in vivo, M26c cells stably transduced with LV-c, LV-shPTCH1 and/or LV- shE2F1 were engrafted subcutaneously into athymic-nude mice. Activation of $\mathrm{HH}$ signaling resulted in almost two-fold larger xenografts than the control cells (Figures 7a, b and c), confirming that the activation of $\mathrm{HH}$ pathway increases melanoma cell growth in vivo. ${ }^{33,58}$ E2F1 silencing reduced}

of about $50 \%$ the size of melanoma xenografts compared with LV-c and strongly reduced the increase of tumor growth induced by PTCH1 silencing (Figures 7a and c). Western blot analysis in tumors dissected 42 days after injection confirmed the increase of E2F1 levels induced by $\mathrm{HH}$ pathway activation and its reduction upon E2F1 silencing (Figure 7d), consistently with the tumor growth curve. The difference in xenograft growth between LV-shE2F1 and LVshPTCH1/LV-shE2F1 was not significant as we documented in vitro. To explain this result, we investigated whether E2F1 depletion in a context of $\mathrm{HH}$ activation might affect the selfrenewal of melanoma CSC grown as spheres. ${ }^{33,59}$ We found that E2F1 silencing markedly reduced the ability of melanoma CSCs to form secondary spheres, suggesting that it might be involved in controlling stemness. The effect of $\mathrm{HH}$ pathway activation was reduced by E2F1 silencing to the level of LV-c. However, even in absence of E2F1, PTCH1 silencing was still able to increase CSC self-renewal, suggesting that the regulation of melanoma stemness by $\mathrm{HH}$ signaling is only partially dependent on E2F1 (Supplementary Figure 13). Nevertheless, western blot analysis on xenografts showed the same iASPP, Cyclin B1 and CDK1 pattern obtained in vitro (Figures $4 d$ and $5 c$ ), suggesting that the HH/GLI-E2F1-iASPP axis contributes to regulate the melanoma cell growth in vivo.

\section{Discussion}

Here we define a novel HH/GLI-E2F1-iASPP axis that regulates melanoma cell growth and identify E2F1 as a central player in determining the outcome of $\mathrm{HH}$ signaling, by controlling the levels and function of the p53 inhibitor iASPP (Figure 8).

Previous data indicated that E2F1 is induced in response to SHH stimulation ${ }^{44,45}$ and by GLI2 overexpression. ${ }^{46}$ Nevertheless, there are no evidence indicating a direct regulation. Here we show that the $\mathrm{HH}$ pathway directly regulates E2F1 expression in melanoma cells and that GLI1 and GLI2 bind to and transactivate the E2F1 promoter. Although bioinformatic analysis did not find any canonical GLI consensus sequence in E2F1 promoter, we identified a functional non-canonical GLI-BS (CGCCTCCAG) by site-directed mutagenesis. This

\footnotetext{
Figure 2 GLI1 and GLI2 bind to E2F1 promoter. (a) Chromatin immunoprecipitation (ChIP) assay showing that both endogenous GLI1 and GLI2 bind to PTCH1 and E2F1 promoters in M26c cells transduced with LV-c or LV-shPTCH1. The y-axis represents the relative promoter enrichment, normalized on the input material. ACTIN promoter was used as negative control and set to 1. (b) Consensus GLI DNA-binding motif calculated from experimentally validated GLI-binding sites ${ }^{50}$ using WebLogo3. ${ }^{65}$ Positions $4 C$ and $6 \mathrm{C}$ are essential for DNA binding, whereas all the other positions allow a certain degree of sequence variation. (c) Quantification of dual-luciferase reporter assay in SSM2c and M26c cells showing that GLI1 and GLI2 transactivate E2F1 promoter in a region between - $132 \mathrm{bp}$ and $-269 \mathrm{bp}$ from the transcription start site. Three fragments of different length $(132,269$ and 656 bp) of E2F1 promoter were assayed for their ability to be transactivated by GLI1 or GLI2; E2F1 was used as a positive control. The red and green arrows mark the positions of the primers used in ChIP, described in Supplementary Figure 4. Relative luciferase activities were firefly/Renilla ratios, with the level induced by control equated to 1. The data represent mean \pm S.E.M. of at least five independent experiments. $P$-values are as follows: -656 bp: CTR versus GLI1, $P=0.0214$ in SSM2c; $P=0.0007$ in M26c; CTR versus GLI2, $P=0.0013$ in SSM2c; $P=0.0182$ in M26c; -269 bp: CTR versus GLI1, $P<0.0001$ in SSM2c; $P=0.0183$ in M26c; CTR versus GLI2, $P<0.0001$ in SSM2c; $P=0.0006$ in M26c; CTR versus E2F1, $P<0.04$ in $-132,-269$ and -656 bp, in both the cells. (d) Putative GLI-binding sites (site1 and site2) in the $-269 \mathrm{bp} E 2 F 1$ promoter that were mutagenized (Mut1 and Mut2). Note that both the positions essential for DNA binding (4C and $6 \mathrm{C}$ ) were mutagenized. (e) Quantification of dual-luciferase reporter assay in SSM2c and M26c cells showing that mutagenesis of Mut1, but not Mut2 site, prevented GLI1 and GLI2 from transactivating the -269 bp fragment of the E2F1 promoter. Relative luciferase activities were firefly/Renilla ratios, with the level induced by the control equated to 1. Data represent mean \pm S.E.M. of at least five independent experiments. (f) Electrophoretic mobility shift assays of site1 in E2F1 promoter. In the right panel the arrow indicates the specific DNA complex that appears upon SAG treatment in NIH3T3 cells. Competition was carried out using a 100-fold excess of unlabeled site1 oligonucleotide. Disruption of the complex is achieved with preincubation of the WCE with anti-Gli1 and anti-Gli2 antibodies. WCE, whole-cell extract; 100x comp, unlabeled site1 competitor. (g) Western blot analysis showing the increase of endogenous Gli1 and Gli2 proteins upon SAG treatment (100 nM, $48 \mathrm{~h}$ ) in NIH3T3 cells. Hsp90 served as loading control
} 
site matches the minimal GLI-BS requirements, ${ }^{50}$ and indeed mutation of two essential cytosines in the 4th and 6th position completely abolishes the ability of GLI1 and GLI2 to transactivate the E2F1 promoter. Despite the divergence from the canonical GLI-BS, EMSA analysis confirmed the binding of GLI1/2 to this site, in agreement with the reporter assay. The biological relevance of this modulation is supported by a significant positive correlation between the expression of E2F1 and of the $\mathrm{HH}$ pathway components (GL/1, GLI2 and $P T C H 1)$ in human melanomas (Figure 1f) and in a set of a

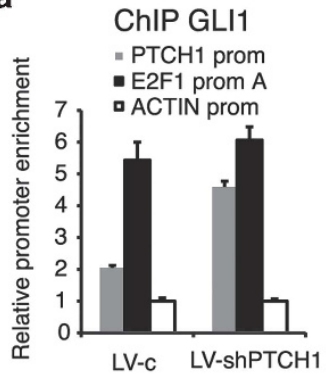

c

ChIP GLI2

b
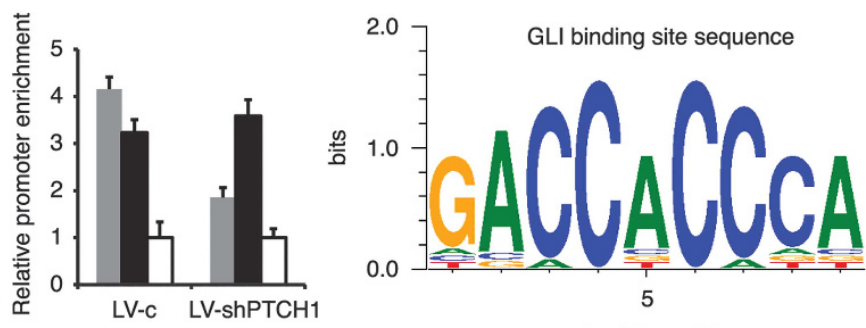

nucleotide position

\section{E2F1 promoter}

$-656 /-1$

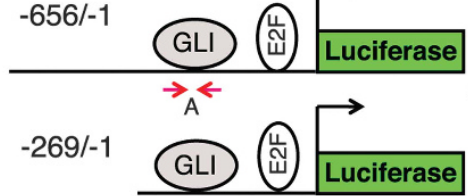

$-132 /-1$
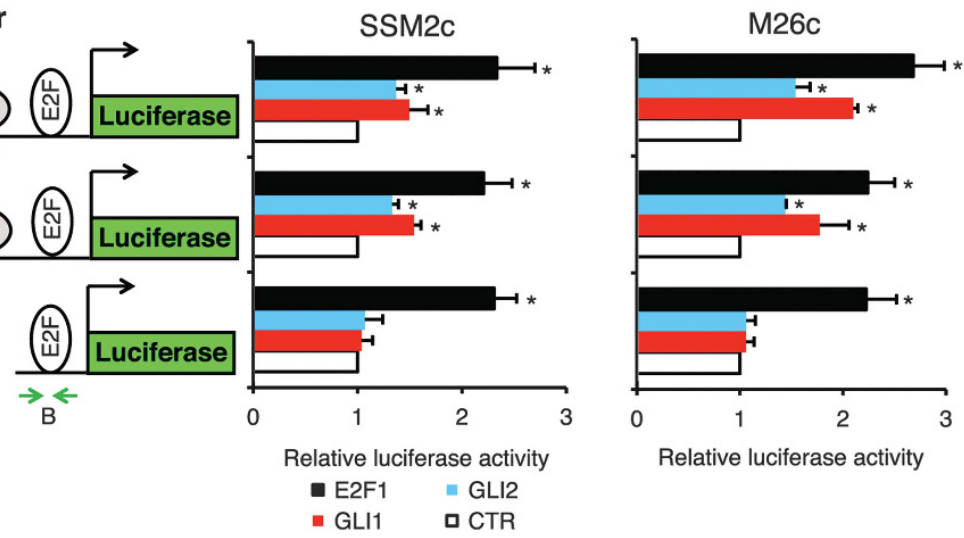

d

E2F1 promoter

Site1

Site2

Wt ATGTTCCGGTGTCCCCACGCCTCCAGC--

CGCATGCAG TACAGTCCC

Mut1

Mut2

e

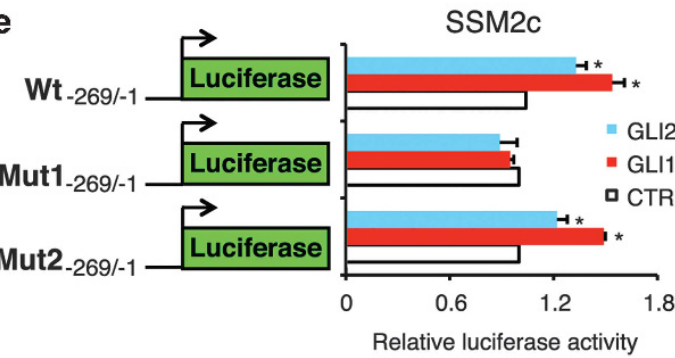

SSM2C

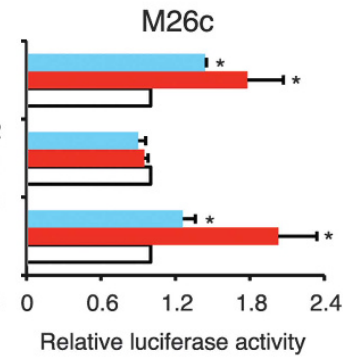

f NIH3T3-SAG NIH3T3+SAG

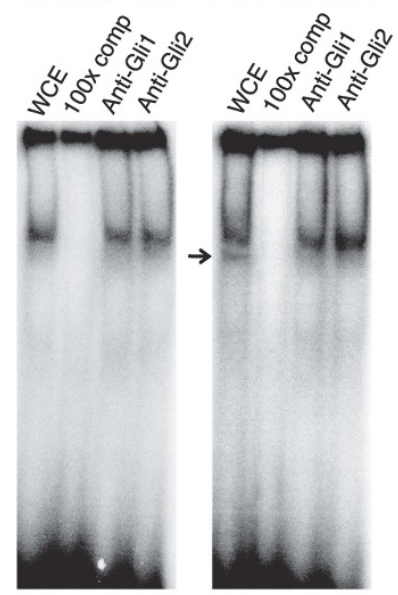

g

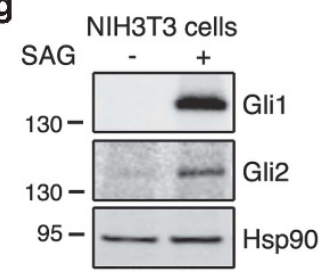



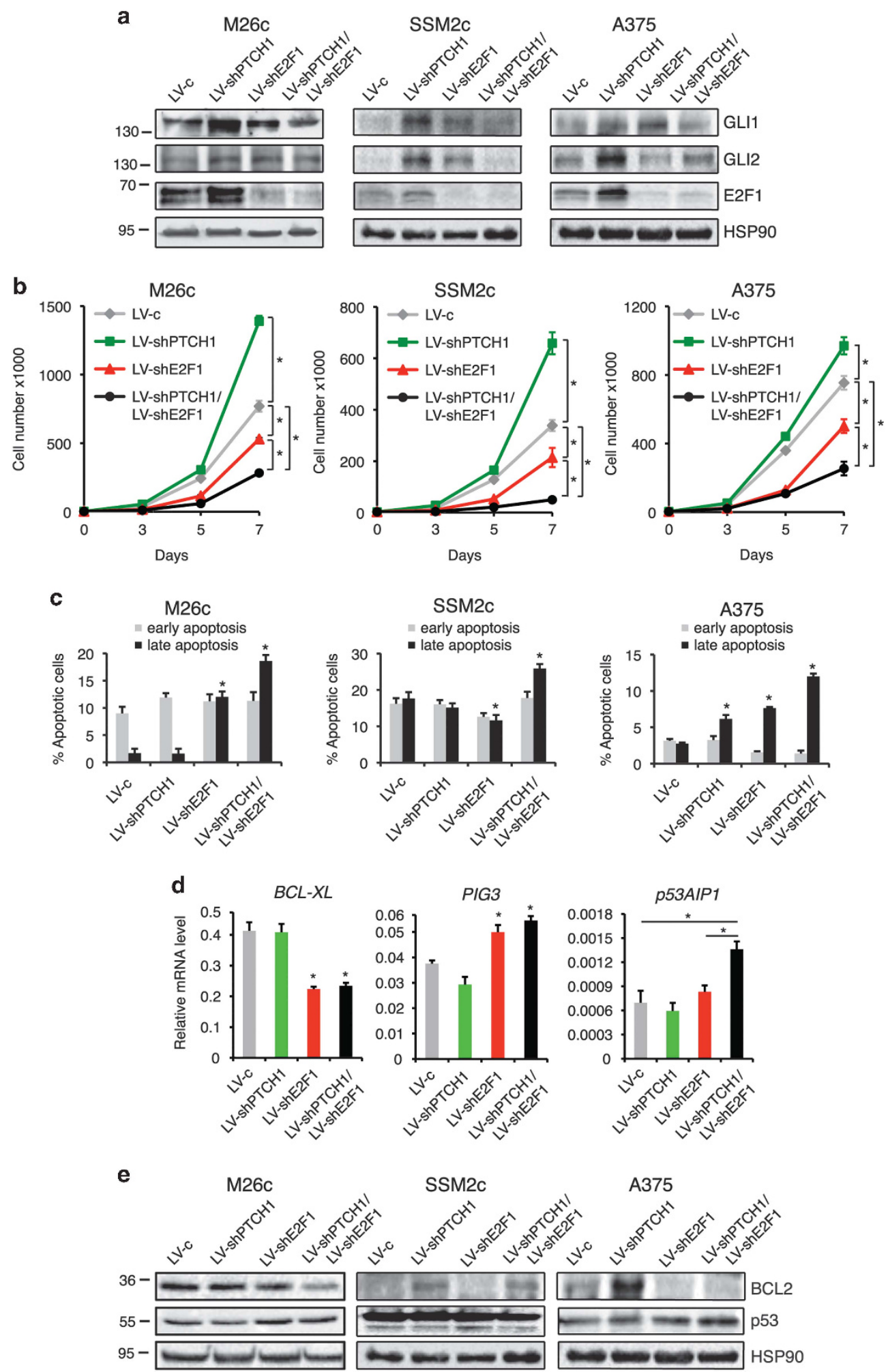

Figure 3 E2F1 is required for the increase in melanoma cell growth induced by activation of the HH signaling. (a) Western blot analysis of GLI1, GLI2 and E2F1 in M26c, SSM2C and A375 cells transduced with the indicated lentiviruses. HSP90 served as loading control. (b) Growth curve in M26c, SSM2C and A375 cells transduced with the indicated lentiviruses, showing that E2F1 silencing abolishes melanoma cell growth induced by activation of the HH pathway. The data represent mean \pm S.E.M. of three independent experiments. ${ }^{*} P<0.05$. (c) Annexin V-PE/7-AAD labeling of M26c, SSM2c and A375 cells transduced with the indicated lentiviruses, showing a marked increase of the late-apoptotic fraction in cells transduced with LV-shE2F1 and with LV-shPTCH1/LV-shE2F1 lentiviruses compared with the control (LV-c). No differences in the early apoptosis are observed. The data represent mean \pm S.E.M. of three independent experiments. ${ }^{*} P<0.05$. (d) Quantitative PCR analysis of $B C L-X L$, PIG3 and $p 53 A I P 1$ expression in M26c cells transduced with the indicated lentiviruses. The $y$-axis represents expression ratio of gene/(EIF2 $\alpha+H P R T$ average). Data represent mean \pm S.E.M. of three independent experiments. ${ }^{*} P<0.05$. (e) Western blot analysis of BCL2 and p53 in M26c, SSM2c and A375 cells transduced with the indicated lentiviruses. HSP90 served as loading control 

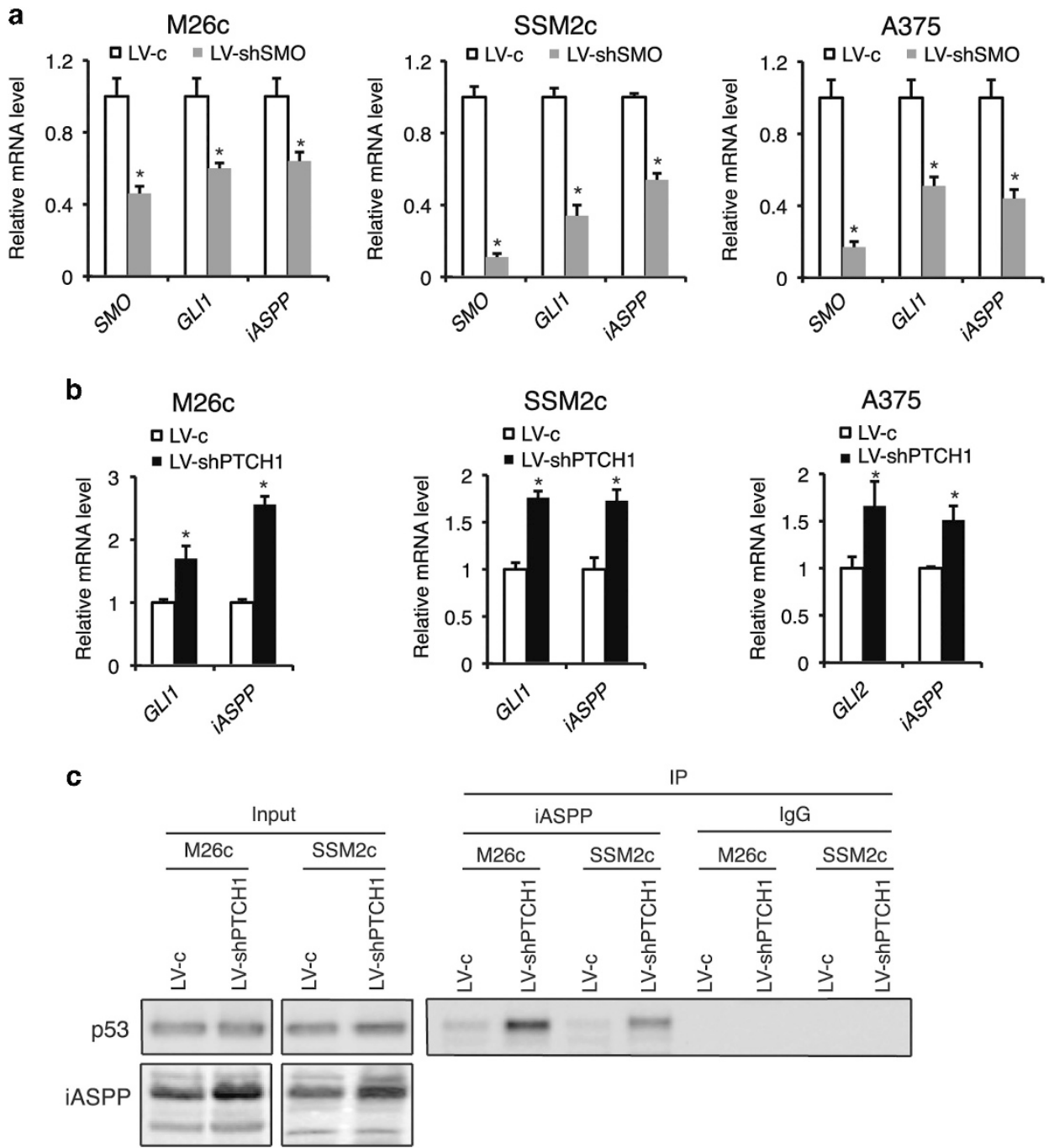

Figure $4 \mathrm{HH}$ pathway modulates iASPP expression and function in melanoma cells. (a) Expression of SMO, GL/1 and iASPP in M26c, SSM2c and A375 cells transduced with LV-c or LV-shSMO lentiviruses, measured by quantitative PCR (qPCR). The $y$-axis represents the expression ratio of gene/(EIF2 $\alpha+H P R T$ average). Data represent mean \pm S.E.M. of three independent experiments. ${ }^{*} P<0.05$. (b) Expression of GLI1 and iASPP in M26c, SSM2c and A375 cells transduced with LV-c or LV-shPTCH1 lentiviruses, measured by qPCR. The $y$-axis represents the expression ratio of gene/(EIF2 $\alpha+H P R T$ average). Data represent mean \pm S.E.M. of three independent experiments. ${ }^{*} P<0.05$. (c) Co-immunoprecipitation to determine the binding between endogenous iASPP and p53 in M26c and SSM2c cells transduced with LV-c or LV-shPTCH1 lentiviruses. Cell lysates are labeled as input

commercial and primary melanoma cells (Supplementary Figure 2).

We previously showed that $\mathrm{HH}$ signaling regulates proliferation and survival of human melanoma and melanoma CSCs. ${ }^{30,33} \mathrm{E} 2 \mathrm{~F} 1$ is a key regulator of cell cycle and survival and it is often upregulated or hyperactivated in a variety of tumors, including melanoma. ${ }^{41,42}$ Our data confirm the role of $\mathrm{HH}$ pathway and E2F1 in regulating melanoma cell growth. Interestingly, the activation of $\mathrm{HH}$ signaling in the absence of E2F1 in vitro produces a marked apoptotic response and reduces melanoma growth more than E2F1 depletion alone. Multiple evidence indicate that activation of $\mathrm{HH}$ signaling leads to the inhibition of the tumor-suppressor p53, ${ }^{34,35}$ which is itself a negative modulator of GLI1. ${ }^{35}$ Although $\mathrm{HH}$ activation promotes cell proliferation, enhanced GLI1 levels are associated with markers of DNA replication stress. ${ }^{35}$ As a consequence, the inhibition of p53 function contributes both to fuel the $\mathrm{HH}$ activation and to blunt the stress response. ${ }^{35}$ Here we identify the HH/GLI-E2F1-iASPP axis as a novel mechanism of p53 inhibition in response to $\mathrm{HH}$ signaling. In this context, E2F1 plays a crucial role in determining the balance between proliferation and apoptosis in response to $\mathrm{HH}$ pathway activation through the regulation of iASPP.

Although iASPP is overexpressed in a variety of human cancers, ${ }^{24-29}$ little is known about the regulation of its expression. Here we find that the activation of $\mathrm{HH}$ signaling increases IASPP expression in melanoma cells. The induction is mediated by E2F1, which binds to the $I A S P P$ promoter in a region closed to a conserved E2F-binding site; notably, the activation of $\mathrm{HH}$ signaling increases E2F1 binding to $I A S P P$ promoter, consistently with the presence of a HH-E2F1-iASPP axis. A previous report showed that the ability of iASPP to inhibit p53 depends on its phosphorylation by Cyclin B1/CDK1 complex..$^{21}$ Our data indicate that $\mathrm{HH}$ signaling contributes to 
a

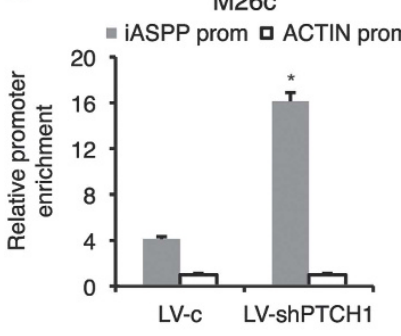

SSM2C

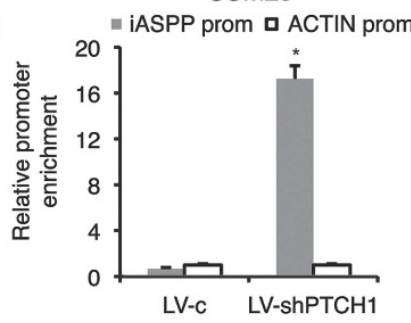

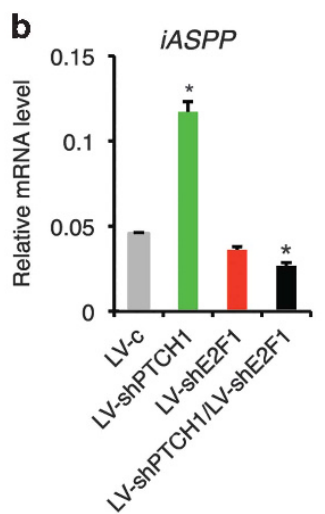

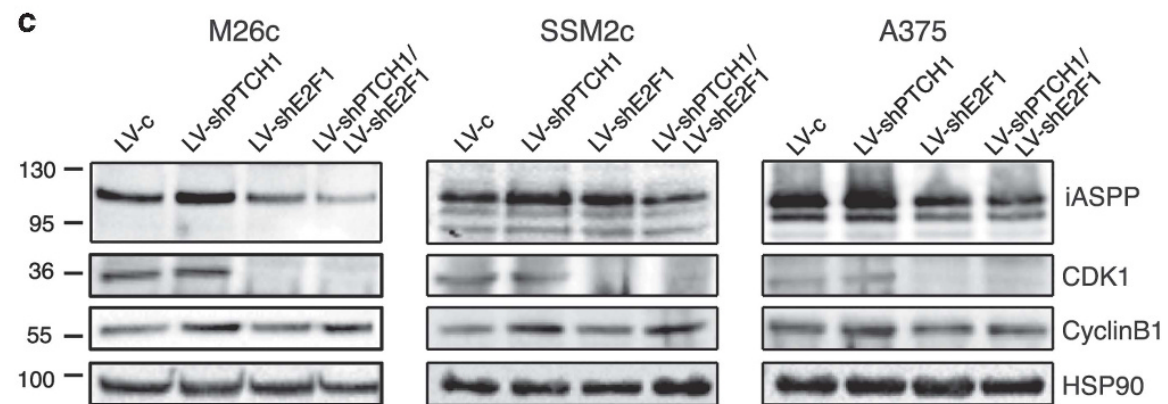

d

M26c

= CDK1 prom $\square$ ACTIN prom

SSM2C

- CDK1 prom $\square$ ACTIN prom
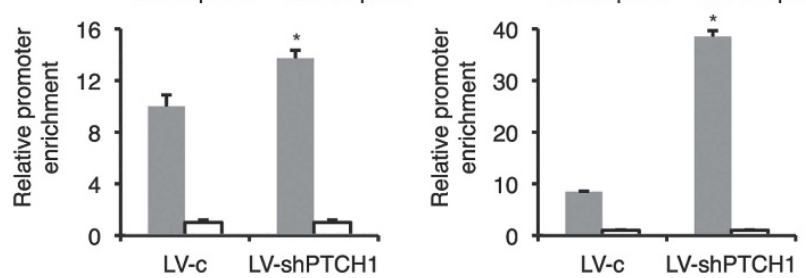

Figure 5 E2F1 mediates the modulation of IASPP by HH pathway in melanoma cells. (a) Chromatin immunoprecipitation (ChIP) assay showing that endogenous E2F1 binds to iASPP promoter in M26c and SSM2c cells transduced with LV-c or LV-shPTCH1. The $y$-axis represents the relative promoter enrichment, normalized on input material. ACTIN promoter was used as negative control and set to 1. (b) Quantitative PCR analysis of iASPP expression in M26c cells transduced with the indicated lentiviruses. The $y$-axis represents the expression ratio of gene/(EIF2 $\alpha+H P R T$ average). Data represent mean \pm S.E.M. of three independent experiments. ${ }^{*} P<0.05$. (c) Western blot analysis of iASPP, CDK1 and Cyclin B1 in M26c, SSM2c and A375 cells transduced with indicated lentiviruses. iASPP was detected using an N-terminal Ab in M26c cells and a C-terminal Ab in SSM2C and A375 cells. HSP90 served as loading control. (d) ChIP assay showing that endogenous E2F1 binds to CDK1 promoter in M26c and SSM2c cells transduced with LV-C or LV-shPTCH1 lentiviruses. The $y$-axis represents the relative promoter enrichment, normalized on input material. ACTIN promoter was used as negative control and set to 1

the regulation of iASPP function also at post-translational level, by controlling both the Cyclin B1 and CDK1 levels and favouring its interaction with $\mathrm{p53}$. Activation of $\mathrm{HH}$ pathway increases Cyclin B1 expression, ${ }^{55,56}$ and this modulation is independent from E2F1. In addition, $\mathrm{HH}$ signaling regulates CDK1 expression through E2F1, ${ }^{53}$ which increases its binding to $C D K 1$ promoter upon $\mathrm{HH}$ signaling activation (Figure 5).

We show that E2F1 is an important mediator of $\mathrm{HH}$ signaling in vivo, because its depletion abolishes the increase in tumor growth induced by the activation of the $\mathrm{HH}$ pathway. We do not find significant difference between LV-shE2F1 and LVshPTCH1/LV-shE2F1 xenografts. This is likely due to other downstream effectors of $\mathrm{HH}$ pathway (e.g. stemness factors) ${ }^{59}$ that in vivo might favor CSC self-renewal independently from E2F1, thus contributing to tumor growth, as suggested by the self-renewal experiments. Nevertheless, as a confirmation of the relevance of our findings, western blot analysis on xenografts reproduces a similar pattern of iASPP, Cyclin B1 and CDK1 expression observed in vitro.

Reactivation of the endogenous wt $\mathrm{p} 53$ has been proposed as a parallel strategy along with targeted therapy to suppress melanoma growth more efficiently. ${ }^{60-62}$ The HH/GLI-E2F1iASPP axis (Figure 8) described in this study provides novel targets for the therapeutic intervention for melanomas and possibly other tumors harboring wt p53 and with activated $\mathrm{HH}$ pathway. Our data show that E2F1 depletion in the context of activated $\mathrm{HH}$ pathway restores p53 apoptotic function. The fact that treatment with JNJ-7706621, a CDK1 inhibitor ${ }^{57}$ that prevents iASPP activation, ${ }^{21}$ produces the same effect in terms of cell growth and apoptosis as E2F1 silencing, confirms the relevance of the $\mathrm{HH}-\mathrm{E} 2 \mathrm{~F} 1$-iASPP axis we show in this study. Therefore, blockade of iASPP activity through interference with E2F1 function or by CDK1 inhibition, is predicted to restore the tumor-suppressive effects of p53, turning the 
a
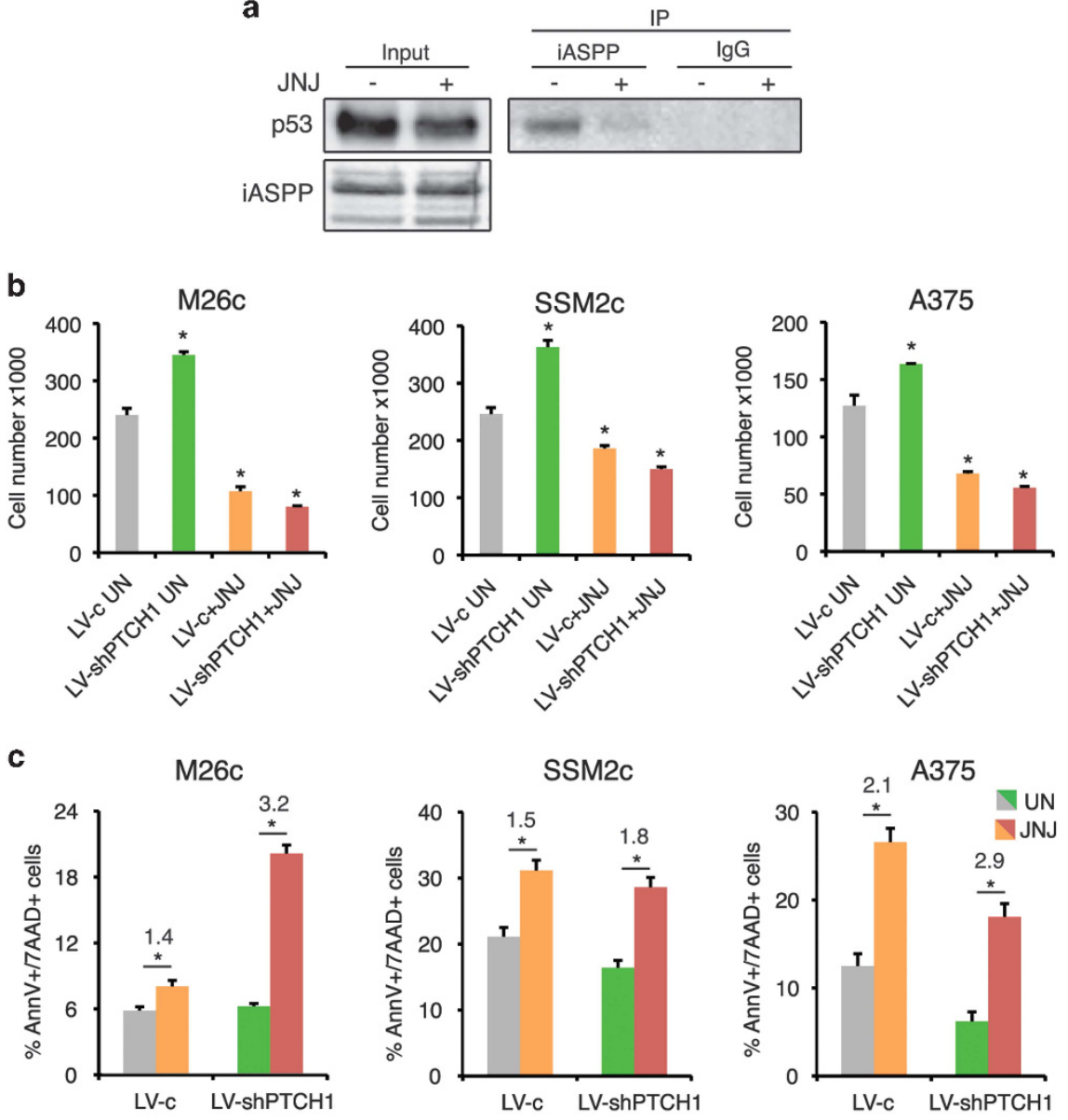

d

M26c
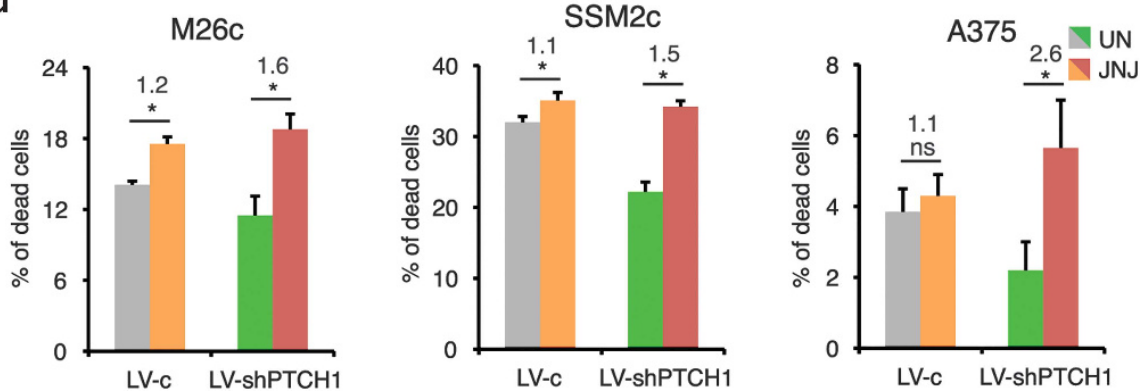

Figure 6 Inhibition of CDK1 mimics silencing of E2F1 upon HH pathway activation. (a) Co-immunoprecipitation to determine the binding between endogenous iASPP and p53 in M26c control cells and treated with $500 \mathrm{nM}$ JNJ-7706621 (JNJ) for $16 \mathrm{~h}$. Cell lysates are labeled as input. (b) Cell growth assay in M26c, SSM2c and A375 cells transduced with LV-c or LV-shPTCH1 lentiviruses after treatment with the CDK1 inhibitor JNJ-7706621 for $72 \mathrm{~h}(500 \mathrm{nM})$. The data represent mean \pm S.E.M. of three independent experiments. ${ }^{*} P<0.05$. (c) Annexin V-PE+/7-AAD+ (late apoptotic) cells in M26c, SSM2c and A375 cells transduced with LV-c or LV-shPTCH1 lentiviruses after treatment with the CDK1 inhibitor JNJ-7706621 for $48 \mathrm{~h}(500 \mathrm{nM})$. The data represent mean \pm S.E.M. of three independent experiments. ${ }^{*} P<0.05$. Fold changes are indicated. (d) Evaluation of cell death by propidium iodide staining of M26c, SSM2c and A375 cells transduced with LV-c or LV-shPTCH1 lentiviruses after treatment with the CDK1 inhibitor JNJ-7706621 for $48 \mathrm{~h}(500 \mathrm{nM})$. The data represent mean \pm S.E.M. of three independent experiments. ${ }^{*} P<0.05$. Fold changes are indicated. UN, untreated cells. Note that cells with activated HH signaling are more sensitive to CDK1 inhibition

proliferative activity of $\mathrm{HH}$ signaling into an apoptotic response.

\section{Materials and methods}

Patient samples and cell cultures. HEK-293T (CRL-11268), NIH3T3 (CRL-1658) and A375 (CRL-1619) cell lines were obtained from ATCC (Manassas, VA, USA). SSM2C and M26c patient-derived melanoma cells were previously described. $^{33,58}$ Human melanoma specimens (Supplementary Table 1) were obtained after approved protocols by the local Ethics Committee. In brief, fresh tissue samples were digested enzymatically using $1 \mathrm{mg} / \mathrm{ml}$ collagenase $A$ and $20 \mu \mathrm{g} / \mathrm{ml}$ DNase I (Roche Diagnostic, Mannheim, Germany) and grown in DMEM supplemented with $10 \%$ fetal bovine serum, $1 \%$ Penicillin-streptomycin, $2 \mathrm{mM}$ L-Glutamine (Lonza, Basel, Switzerland). The identity of melanoma cells was verified by immunocytochemistry using anti-Melan $A$, anti-S100 and anti-Vimentin antibodies, as previously described. ${ }^{33}$ Mycoplasma was periodically tested by 4',6diamidino-2-phenylindole inspection and PCR. Direct sequencing revealed that both SSM2c and M26c cells harbor wt p53 with codon 72 Proline polymorphism. ${ }^{58}$ A375 

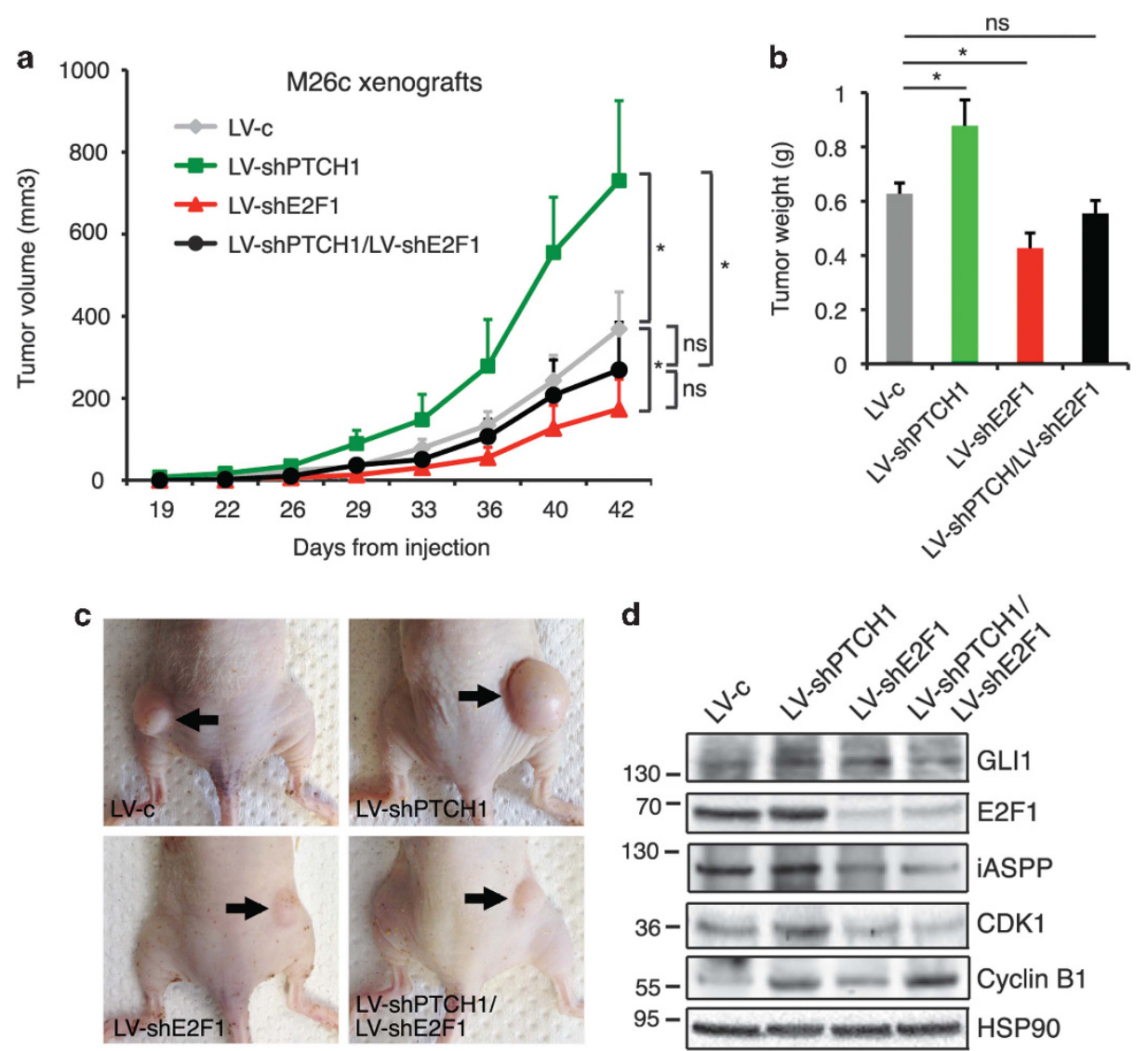

Figure 7 E2F1 silencing prevents HH-induced melanoma xenograft growth. (a) M26c cells transduced with LV-c, LV-shPTCH1, LV-shE2F1 or LV-shPTCH1/LV-shE2F1 lentiviruses were injected subcutaneously in athymic-nude mice. Quantification of tumor volume ( $n=12$ per group), showing that E2F1 silencing prevented the increase of tumor growth induced by the activation of the HH signaling. ${ }^{*} P<0.05$. (b) Tumor weight of xenografts, as indicated. ${ }^{*} P<0.05$. (c) Representative images of M26c xenografts, as indicated. (d) Western blot analysis of GLI1, E2F1, iASPP, CDK1 and Cyclin B1 in tumors derived from M26c xenografts. iASPP was detected using an N-terminal Ab. HSP90 served as loading control

melanoma cells harbor wt p53. For growth curve 3000 cells/well were plated in 12well plates and counted on days 3-5-7. For growth assay after JNJ treatment 15000 cells/well were plated in 12-well plates and treated with the CDK1 inhibitor JNJ-7706621 (500 nM, JNJ; Merck, Damstadt, Germany) for $72 \mathrm{~h}$. Puromycin was used at $2 \mu \mathrm{g} / \mathrm{ml}$ to select for transduced cells.

Plasmids, cloning, mutagenesis and lentiviral vectors. Vectors used for overexpression were: pCS2+MT (Addgene, Cambridge, MA, USA), pCMVE2F1 (kind gift from M. Chiariello), ${ }^{63}$ Myc-tagged human GLI1 (pCS2+MT-GLI1, kind gift from A. Ruiz i Altaba) ${ }^{35}$ and GLI2 (pCS2+MT-GLI2, Addgene). ${ }^{5}$ Three fragments of E2F1 promoter $(-132,-269$ and $-656 \mathrm{bp}$ ) were PCR amplified with Platinum Pfx DNA polymerase (Life Technologies, Carlsbad, CA, USA) and cloned in pGL3Basic vector (Promega, Madison, WI, USA) using Nhel-Xhol sites, to generate $-132 /-269 /-656$ bp-E2F1 prom-luc reporters. Primers used were: E2F1 prom - 132 bp, FW, 5'-ACGCTAGCGCGCGTTAAAGCCAATAGG-3'; E2F1 prom -269 bp, FW, 5'-ACGCTAGCATGTTCCGGTGTCCCCAC-3'; E2F1 prom -656 bp, FW, 5'-ACGCTAGCTGAGTGAGGGAATGAATG-3'; E2F1 prom, RV, 5'-ACCTCGAGATC CTTTTTGCCGCGAAA-3'. Mutations of E2F1 prom - 269-bp reporter were introduced using QuickChange II (Agilent Technologies, Santa Clara, CA, USA) with the following oligos: Mut1, FW, 5'-GGTGTCCCCACGCATGCAGCCAGGGGACG-3'; Mut1, RV, 5'-CGTCCCCTGGCTGCATGCGTGGGGACACC-3'; Mut2, FW, 5'-GCCA TTGGCCGTACAGTCCCGCGCCGCCGCC-3'; Mut2, RV, 5'-GGCGGCGGCGCGG GACTGTACGGCCAATGGC-3'. Plasmid identity was verified by direct sequencing. All transfections were performed in OptiMEM (Life Technologies) using $X$-tremeGENE transfection reagent (Roche Diagnostic) according to the manufacturer's protocol. Lentiviruses were produced in HEK-293T cells. Lentiviral vectors pLV-CTH (LV-c), pLV-CTH-shPTCH1 (LV-shPTCH1) ${ }^{35}$ and pLKO.1-puro-

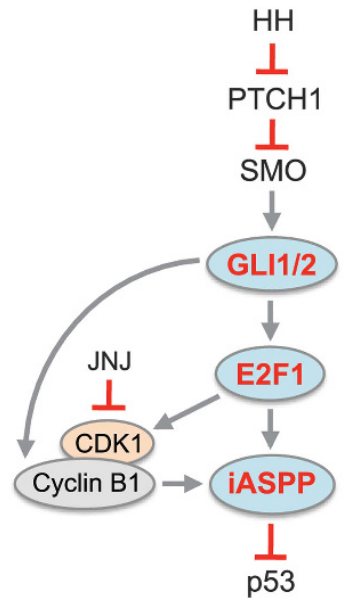

Figure 8 Model of the HH/GLI-E2F1-iASPP axis. E2F1 is a direct target of GLI1 and GLI2, and acts downstream of $\mathrm{HH}$ signaling by controlling the expression and activation of IASPP. E2F1 regulates iASPP expression and CDK1 expression. The effectors of HH signaling GLI1 and GLI2 regulate Cyclin B1 expression. The complex CDK1/Cyclin B1 phosphorylates iASPP and increases its ability to bind and inactivate p53. JNJ-7706621 (JNJ) is a CDK1 inhibitor 
shSMO (LV-shSMO) ${ }^{33}$ were previously described. pLKO.1-puro (LV-c), pLKO.1-puroshE2F1-1 (LV-shE2F1-1; targeting sequence 5'- GACCTCTTCGACTGTGACTTT-3', exon 7) were from Open Biosystems (Lafayette, CO, USA). Most experiments were performed with LV-shE2F1-1.

Luciferase reporter assay. E2F1 prom-luc reporters (-132/-269/ $-656 \mathrm{bp}$ ) were used in combination with Renilla luciferase $\mathrm{pRL}-\mathrm{TK}$ reporter vector (Promega) to normalize luciferase activities; pGL3Basic vector (Promega) was used to equal DNA amounts. Luminescence was measured using the Dual-Glo Luciferase Assay System (Promega) and the GloMax 20/20 Luminometer (Promega).

Electrophoretic mobility shift assay. NIH3T3 cells were treated with $100 \mathrm{nM}$ SAG for $48 \mathrm{~h}$ to activate the HH pathway, harvested and lysed in lysis buffer (20 mM HEPES pH 7.9, 25\% glycerol, 0.5\% Nonidet $P-40,420 \mathrm{mM} \mathrm{NaCl}, 1.5 \mathrm{mM}$ $\mathrm{MgCl}_{2}, 0.2 \mathrm{mM}$ EDTA, $1 \mathrm{mM}$ DTT) to obtain whole-cell extracts (WCE). ${ }^{64}$ To analyze the binding of Gli proteins to Site1 at E2F1 promoter we used the following oligonucleotide and its complementary strand: site1F, $5^{\prime}$-AGCTACCCTGGAG GCGTCT-3'; site1R, 5'-TCGAAGACGCCTCCAGGGT-3'. The DNA-binding reaction was performed by incubating $20 \mathrm{fmol}$ of double-stranded ${ }^{32} \mathrm{P}$-labeled oligo with $40 \mu \mathrm{g}$ of WCE in a total volume of $20 \mu \mathrm{l}$ containing $1 \mu \mathrm{g}$ poly-dC, $100 \mathrm{mM} \mathrm{KCl}$ in $40 \mathrm{mM}$ HEPES (pH 7.9), $10 \mathrm{mM} \mathrm{MgCl2,} 0.4 \mathrm{mM}$ EDTA, $4 \mathrm{mM}$ DTT and $40 \%$ glycerol ${ }^{64}$ for $20 \mathrm{~min}$ at room temperature. In indicated competition experiments, a 100-fold excess of unlabeled oligo was used. When using antibodies, proteins were preincubated with anti-GLI1 (N-16, Santa Cruz Biotechnology, Santa Cruz, CA, USA) or anti-GLI2 (\#AF3635, R\&D Systems, Minneapolis, MN, USA) antibodies for $20 \mathrm{~min}$ at room temperature before adding the radiolabeled probe. The samples were separated on native polyacrylamide gel $(6 \%$ polyacrylamide:bisacrylamide, 29:1) at $4{ }^{\circ} \mathrm{C}$ for $3 \mathrm{~h}$ and the signal was detected by Cyclone Storage Phosphor System (Perkin Elmer, Waltham, MA, USA).

Western blot and co-immunoprecipitation. Samples were lysed in ice in RIPA buffer ( $1 \% \mathrm{NP}-40,150 \mathrm{mM} \mathrm{NaCl}, 5 \mathrm{mM}$ EDTA, $0.25 \% \mathrm{NaDOC}, 50 \mathrm{mM}$ Tris- $\mathrm{HCl} \mathrm{pH} 7.5$, SDS $0,1 \%$ ) added with protease and phosphatase inhibitors, sonicated to shear DNA and centrifuged at 14000 r.p.m. for 20 min at $4{ }^{\circ} \mathrm{C}$; supernatant was collected as WCE. For co-immunoprecipitation experiments $700 \mu \mathrm{g}$ WCE were diluted with IP buffer $(0.5 \% \mathrm{NP}-40,100 \mathrm{mM} \mathrm{NaCl}, 5 \mathrm{mM}$ EDTA, $10 \%$ glycerol, $50 \mathrm{mM}$ Tris- $\mathrm{HCl} \mathrm{pH} 7.5$ ) added with protease and phosphatase inhibitors to a final volume of $450 \mu$ and incubated overnight at $4{ }^{\circ} \mathrm{C}$ with Dynabeads Protein $\mathrm{G}$ (Life Technologies) pre-conjugated with anti-iASPP antibody (49.3, Santa Cruz Biotechnology) or irrelevant IgG (Life Technologies). Beads were washed three times with IP buffer, proteins were eluted with Laemmli buffer and visualized on SDS polyacrylamide gel electrophoresis. The following antibodies were used for western blot: rabbit anti-iASPP (ab34898), (Abcam, Cambridge, United Kingdom), rabbit anti-E2F1 (\#3742), rabbit anti-BCL2 (\#2976), mouse antiGLI1 (L42B10) (Cell Signaling Technology, Danvers, MA, USA), goat anti-GLI2 (\#AF3635; R\&D Systems), mouse anti-Myc (9E10), mouse anti-HSP90 (F-8), mouse anti-p53 (DO-1), mouse anti-iASPP (2808C5a), rabbit anti-CDK1 (C-19), rabbit anti-Cyclin B1 (H-433; Santa Cruz Biotechnology), mouse anti- $\beta$-ACTIN (AC-15; Sigma-Aldrich, St. Louis, MO, USA). Chemiluminescent detection was used.

Quantitative real-time PCR. Total RNA from adherent cells was isolated with TriPure Isolation Reagent (Roche Diagnostics). After DNase I treatment (Roche Diagnostics), RNA was subjected to reverse transcription with High Capacity cDNA Reverse Transcription Kit (Life Technologies). qPCRs were carried out at $60^{\circ} \mathrm{C}$ using FastStart SYBR Green Master (Roche Diagnostic) in a Rotorgene-Q (Qiagen, Hilden, Germany). Primer sequences are the following ( $5^{\prime}$ to $\left.3^{\prime}\right)$ : GLI1-F, 5'-CCCAGTACATGCTGGTGGTT-3'- and GLI1-R, 5'-GCTTTACTGCAGCCCTCGT-3'; PTCH1-F, 5'-GGCAGCGGTAGTAGTGGTGTTC-3'- and PTCH1-R, 5'-TGTAGC GGGTATTGTCGTGTGTG-3'-; SMO-F 5'-GGGAGGCTACTTCCTCATCC-3'- and SMO-R 5'-GGCAGCTGAAGGTAATGAGC-3'-; E2F1-F, 5'-GCTGAGCCACTCGG CTGACG-3'-; E2F1-R, 5'-CACTGTGGTGTGGCTGCCC-3'-; iASPP-F, 5'-AGCCT TAAAGAGACAGGACGG-3'; iASPP-R, 5'-TGTTTCATGGCCAGCGACT-3'; BCLXL-F, 5'-GGTAAACTGGGGTCGCATTG-3'; BCL-XL-R, 5'-GCTGCTGCATTGTT CCCATAG-3'; PIG3-F, 5'-CTGAACCGGGCGGACTTAAT-3'; PIG3-R, 5'-GTGTCCC CGATCTTCCAGTG-3'; p53AIP1-F, 5'-CTCGGTGATGCCTCCGAATG-3'; p53AIP1-R, $5^{\prime}$-GGCCTGGAGAGACCTAGACC-3'. Primers for human reference genes are: EIF2a-F, 5'- GGATGGGACCTTGTTTGCCT-3' and EIF2a-R, 5'-CCACGTTGCCA
GGACAGTAT-3'; HPRT-F, 5'- GCCAGACTTTGTTGGATTTG-3' and HPRT-R, 5'-CTCTCATCTTAGGCTTTGTATTTTG-3'.

Chromatin immunoprecipitation. Melanoma cells were fixed with $1 \%$ formaldehyde for $10 \mathrm{~min}$ and fixation was stopped by adding $125 \mathrm{mM}$ glycine for $5 \mathrm{~min}$. Cells were harvested and lysed in Cell Lysis Buffer ( $5 \mathrm{mM}$ PIPES pH 8, $85 \mathrm{mM} \mathrm{KCl}, 0.5 \% \mathrm{NP}-40$ ) added with protease inhibitors. Nuclei were collected by centrifugation and lysated in nuclear lysis buffer (1\% SDS, $10 \mathrm{mM}$ EDTA, $50 \mathrm{mM}$ Tris- $\mathrm{HCl} \mathrm{pH} \mathrm{8)} \mathrm{added} \mathrm{with} \mathrm{protease} \mathrm{inhibitors.} \mathrm{Chromatin} \mathrm{was} \mathrm{sonicated} \mathrm{to} \mathrm{an}$ average size of $300-500 \mathrm{bp}$ using a SONOPULS Mini20 Sonicator (Bandelin, Berlin, Germany) equipped with a cuphorn and diluted with ChIP Dilution Buffer (1.8\% Triton X-100, $2 \mathrm{mM}$ EDTA, $300 \mathrm{mM} \mathrm{NaCl}$ ); input material was collected. Chromatin was incubated overnight with Dynabeads Protein G (Life Technologies) pre-conjugated with anti-GLI1 (N-16; Santa Cruz Biotechnology), anti-GLI2 (\#AF3635, R\&D Systems) or anti-E2F1 (\#3742; Cell Signaling Technology) antibodies. Immunocomplexes were washed with low salt wash buffer $(20 \mathrm{mM}$ Tris$\mathrm{HCl}, \mathrm{pH}$ 8, $2 \mathrm{mM}$ EDTA, $150 \mathrm{mM} \mathrm{NaCl}, 0.1 \%$ SDS, $1 \%$ Triton X-100), high salt wash buffer (20 mM Tris-HCl, pH 8, 2 mM EDTA, $500 \mathrm{mM} \mathrm{NaCl}, 0.1 \%$ SDS, $1 \%$ Triton $\mathrm{X}-100$ ) and LiCl wash buffer (250 mM LiCl, 1\% NP-40, 1 mM EDTA, $10 \mathrm{mM}$ Tris-HCl, $\mathrm{pH} 8,1 \%$ sodium deoxycholate) and twice with TE (1 mM EDTA, $10 \mathrm{mM}$ Tris-HCl, $\mathrm{pH}$ 8). All the solutions were added with $1 \mathrm{X}$ complete Protease Inhibitor Cocktail (Roche Diagnostic). DNA was eluted with $1 \% \mathrm{SDS}$ at $85^{\circ} \mathrm{C}$ for $10 \mathrm{~min}$, crosslinks were reversed overnight at $65^{\circ} \mathrm{C}$ with $200 \mathrm{mM} \mathrm{NaCl}$. After treatment with $4 \mu \mathrm{g}$ RNaseA (Life Technologies) at $37^{\circ} \mathrm{C}$ for $30 \mathrm{~min}$ and with $20 \mu \mathrm{g}$ Proteinase $\mathrm{K}$ (Roche Diagnostic) at $60^{\circ} \mathrm{C}$ for $2 \mathrm{~h}$, the DNA was recovered by using the QIAquick PCR Purification Kit (Qiagen). qPCR were carried out at $60^{\circ} \mathrm{C}$ using FastStart SYBR Green Master (Roche Diagnostic) in a Rotorgene-Q (Qiagen). Primers used were: E2F1promA-F, 5'-CTATAGAAAGGTCAGTGGGATGC-3' and E2F1promA-R, 5'-AGGCTTTGTCCGGATGGTA-3'; PTCH1prom-F, 5'-ACACACTGGGTTGCCTACC-3' and PTCH1prom-R, 5'-CTGTCAGATGGCTTGGGTTT-3'; iASPPprom-F, 5'-GGAG AAATAGGGGCAATCCGT-3' and IASPPprom-R, 5'-GCATGAGACTAAACCCCCGA-3'; CDK1prom-F, 5'-TCGCTCTCCGCTCAATTTCC-3' and CDK1prom-R, 5'-GGGCT ACCCGATTGGTGAAT-3'; ACTINprom-F, 5'-TCGAGCCATAAAAGGCAACT-3' and ACTINprom-R, 5'-CTTCCTCAATCTCGCTCTCG-3'.

Flow cytometry analysis. Apoptosis was assessed by cytometric analysis of Annexin V/7-AAD labeled cells using Annexin V-PE/7-AAD apoptosis kit (Becton Dickinson, Franklin Lakes, NJ, USA), according to manifacturer's protocol. Cell death was measured by propidium iodide staining; cells were incubated with $100 \mathrm{ng} / \mathrm{ml} \mathrm{PI}$ for $5 \mathrm{~min}$ before analysis. Cytometric analysis was performed with FACS-Canto II (Becton Dickinson).

Cell sorting, nude mice and xenografts. Melanoma cells were transduced with either pLV-CTH (LV-c) or pLV-CTH-shPTCH1 (LV-shPTCH1) lentiviruses. Cells transduced with LV-c or LV-shPTCH1 also express green fluorescent protein (GFP) and were FACS (fluorescent-activated cell sorter)-sorted with the BD FACS-Aria cell sorter (Becton Dickinson). GFP-positive cells were then transduced with either pLKO.1-puro (LV-c) or pLKO.1-puro-shE2F1-1 (LV-shE2F1) lentiviruses and selected for puromycin resistance. For xenotransplantation M26C cells were resuspended in Matrigel (Becton Dickinson) /DMEM (1/1) and inoculated subcutaneously in lateral flanks of adult female athymic-nude mice (Foxn1 nu/nu; Harlan Laboratories, Udine, Italy; 40000 cells/injection). Animals were housed in SPF conditions and monitored daily. Subcutaneous tumor size was measured twice a week with a caliper. Tumor volumes were calculated using the formula: $V=W^{2} \times L$ $x$ 0.5, where $W$ and $L$ are, respectively, tumor width and length. The experiment was approved by the Italian Ministry of Health and was in accordance with the Italian guidelines and regulations.

Statistical analysis. The data represent mean \pm S.E.M. values and are calculated on at least $3-5$ independent experiments. $P$-values were calculated using Student's $t$-test. A two-tailed value of $P<0.05$ was considered statistically significant.

Bioinformatic analysis. Publicly available gene expression data for a series of 31 primary and 73 metastatic melanomas were profiled on Affymetrix U133 platform (Gene Expression Omnibus GEO-46517). ${ }^{47}$ To assess the relationship between E2F1, PTCH1, GLI1 and GLI2 expression we performed Pearson's correlation and simple regression analysis using StatGraphics Centurion XV.I software (Statpoint Technologies). 


\section{Conflict of Interest}

The authors declare no conflict of interest.

Acknowledgements. We thank Gianni Del Sal (ICGEB, Trieste, Italy), Silvia Pietrobono, Roberta Santini, Maria Riverso and Alessandro Apollo (Tumor Cell Biology Unit, ITT, Florence) for helpful comments on the paper and discussion. We are grateful to Lorenzo Borgognoni, Gianni Gerlini (S. Maria Annunziata Hospital, Florence, Italy), Nicola Pimpinelli (Department of Dermatology, University of Florence, Florence, Italy) and Riccardo Gattai (Department of Medical-Surgical Critical Area, General and Oncological Surgery, University of Florence, Florence, Italy) for providing samples and Laura Poliseno (Istituto Toscano Tumori, Pisa, Italy) for providing SKMel-2, SK-Mel-5, SK-Mel-28 and 501Mel melanoma cell lines. This work was supported by grants from AIRC (Associazione Italiana per la Ricerca sul Cancro; IG-9566 and IG-14184), Regional Health Research Program 2009 and Fondazione Cassa di Risparmio di Firenze (2011.1072, FiorGen Foundation) to BS.

1. Ingham PW, McMahon AP. Hedgehog signaling in animal development: paradigms and principles. Genes Dev 2002; 15: 3059-3087.

2. Ruiz i Altaba A Hedgehog-Gli signaling in Human Diseases. Plenum, 2006.

3. Chiang C, Litingtung Y, Lee E, Young KE, Corden JL, Westphal $\mathrm{H}$ et al. Cyclopia and defective axial patterning in mice lacking Sonic hedgehog gene function. Nature 1996; 383 : 407-413.

4. Belloni E, Muenke M, Roessler E, Traverso G, Siegel-Bartelt J, Frumkin A et al. Identification of Sonic hedgehog as a candidate gene responsible for holoprosencephaly. Nat Genet 1996; 14: 353-356.

5. Roessler E, Ermilov AN, Grange DK, Wang A, Grachtchouk M, Dlugosz AA et al. A previously unidentified amino-terminal domain regulates transcriptional activity of wild-type and disease-associated human GLI2. Hum Mol Genet 2005; 14: 2181-2188.

6. Beachy PA, Karhadkar SS, Berman DM. Tissue repair and stem cell renewal in carcinogenesis. Nature 2004; 432: 324-331.

7. Teglund S, Toftgård R. Hedgehog beyond medulloblastoma and basal cell carcinoma. Biochim Biophys Acta 2010; 1805: 181-208.

8. Pandolfi S, Stecca B. Cooperative integration between HEDGEHOG-GLI signalling and other oncogenic pathways: implications for cancer therapy. Expert Rev Mol Med 2015; 17 : e5.

9. Rohatgi R, Scott MP. Patching the gaps in Hedgehog signalling. Nat Cell Biol 2007; 9 : 1005-1009.

10. Jiang J, Hui CC. Hedgehog signaling in development and cancer. Dev Cell 2008; 15 : 801-812.

11. Oliver TG, Grasfeder LL, Carroll AL, Kaiser C, Gillingham CL, Lin SM et al. Transcriptional profiling of the Sonic hedgehog response: a critical role for $\mathrm{N}$-myc in proliferation of neuronal precursors. Proc Natl Acad Sci USA 2003; 100: 7331-7336.

12. Milla LA, Arros A, Espinoza N, Remke M, Kool M, Taylor MD et al. Neogenin1 is a Sonic Hedgehog target in medulloblastoma and is necessary for cell cycle progression. Int J Cancer 2014; 134: 21-31.

13. Balch CM, Gershenwald JE, Soong SJ, Thompson JF, Atkins MB, Byrd DR et al. Final version of 2009 AJCC melanoma staging and classification. J Clin Oncol 2009; 27: 6199-6206.

14. Straume O, Akslen LA. Alterations and prognostic significance of p16 and p53 protein expression in subgroups of cutaneous melanoma. Int J Cancer 1997; 74: 535-539.

15. Hodis E, Watson IR, Kryukov GV, Arold ST, Imielinski M, Theurillat JP et al. A landscape of driver mutations in melanoma. Cell 2012; 150: 251-263.

16. Curtin JA, Fridlyand J, Kageshita T, Patel HN, Busam KJ, Kutzner $\mathrm{H}$ et al. Distinct sets of genetic alterations in melanoma. N Engl J Med 2005; 353: 2135-2147.

17. Zhang $Y$, Xiong $Y$, Yarbrough WG. ARF promotes MDM2 degradation and stabilizes $p 53$ : ARF-INK4a locus deletion impairs both the Rb and p53 tumor suppression pathways. Cell 1998; 92: 725-734.

18. Gembarska A, Luciani F, Fedele C, Russell EA, Dewaele M, Villar S et al. MDM4 is a key therapeutic target in cutaneous melanoma. Nat Med 2012; 18: 1239-1247.

19. Muthusamy V, Hobbs C, Nogueira C, Cordon-Cardo C, McKee PH, Chin L et al. Amplification of CDK4 and MDM2 in malignant melanoma. Genes Chromosomes Cancer 2006; 45: 447-454.

20. Polsky D, Melzer K, Hazan C, Panageas KS, Busam K, Drobnjak M et al. HDM2 protein overexpression and prognosis in primary malignant melanoma. J Natl Cancer Inst 2002; 94: 1803-1806.

21. Lu M, Breyssens $\mathrm{H}$, Salter V, Zhong S, Hu Y, Baer $\mathrm{C}$ et al. Restoring p53 function in human melanoma cells by inhibiting MDM2 and cyclin B1/CDK1-phosphorylated nuclear IASPP. Cancer Cell 2013; 23: 618-633.

22. Bergamaschi D, Samuels Y, O'Neil NJ, Trigiante G, Crook T, Hsieh JK et al. 2003 iASPP oncoprotein is a key inhibitor of p53 conserved from worm to human. Nat Genet 2003; 33: 162-167.
23. Bergamaschi D, Samuels $Y$, Sullivan A, Zvelebil M, Breyssens H, Bisso A et al. iASPP preferentially binds p53 proline-rich region and modulates apoptotic function of codon 72-polymorphic p53. Nat Genet 2006; 38: 1133-1141.

24. Zhang X, Wang M, Zhou C, Chen S, Wang J.. The expression of iASPP in acute leukemias. Leuk Res 2005; 29: 179-183.

25. Saebø M, Skjelbred CF, Nexø BA, Wallin H, Hansteen IL, Vogel U et al. Increased mRNA expression levels of ERCC1, OGG1 and RAI in colorectal adenomas and carcinomas. BMC Cancer 2006; 6: 208-214.

26. Liu Z, Zhang X, Huang D, Liu Y, Zhang X, Liu L et al. Elevated expression of IASPP in head and neck squamous cell carcinoma and its clinical significance. Med Oncol 2012; 29: $3381-3388$

27. Chen J, Xie F, Zhang L, Jiang WG. iASPP is over-expressed in human non-small cell lung cancer and regulates the proliferation of lung cancer cells through a p53 associated pathway. BMC Cancer 2010; 10: 694

28. Cao L, Huang Q, He J, Lu J, Xiong Y. Elevated expression of iASPP correlates with poor prognosis and chemoresistance/radioresistance in FIGO Ib1-lla squamous cell cervical cancer. Cell Tissue Res 2013; 352: 361-369.

29. Morris EV, Cerundolo L, Lu M, Verrill C, Fritzsche F, White MJ et al. Nuclear iASPP may facilitate prostate cancer progression. Cell Death Dis 2014; 5: e1492.

30. Stecca B, Mas C, Clement V, Zbinden M, Correa R, Piguet V et al. Melanomas require HEDGEHOG-GLI signaling regulated by interactions between GLI1 and the RAS-MEK/AKT pathways. Proc Natl Acad Sci USA 2007; 104: 5895-5900.

31. Jalili A, Mertz KD, Romanov J, Wagner C, Kalthoff F, Stuetz A et al. NVP-LDE225, a potent and selective SMOOTHENED antagonist reduces melanoma growth in vitro and in vivo. PLoS One 2013; 8: e69064.

32. O'Reilly KE, de Miera EV, Segura MF, Friedman E, Poliseno L, Han SW et al. Hedgehog pathway blockade inhibits melanoma cell growth in vitro and in vivo. Pharmaceuticals 2013; 6: $1429-1450$.

33. Santini R, Vinci MC, Pandolfi S, Penachioni JY, Montagnani V, Olivito B et al. Hedgehog-GLI signaling drives self-renewal and tumorigenicity of human melanoma-initiating cells. Stem Cells 2012; 30: 1808-1818.

34. Abe Y, Oda-Sato E, Tobiume K, Kawauchi K, Taya Y, Okamoto K et al. Hedgehog signaling overrides p53-mediated tumor suppression by activating Mdm2. Proc Natl Acad Sci USA 2008; 105: 4838-4843.

35. Stecca B, Ruiz i Altaba A. A GLI1-p53 inhibitory loop controls neural stem cell and tumour cell numbers. EMBO J 2009; 28: 663-676.

36. Mazzà D, Infante P, Colicchia V, Greco A, Alfonsi R, Siler M et al. PCAF ubiquitin ligase activity inhibits Hedgehog/Gli1 signaling in p53-dependent response to genotoxic stress. Cell Death Differ 2013; 20: 1688-1697.

37. Verhaegen M, Checinska A, Riblett MB, Wang S, Soengas MS. E2F1-dependent oncogenic addiction of melanoma cells to MDM2. Oncogene 2012; 31: 828-841.

38. Huang Y, Ishiko T, Nakada S, Utsugisawa T, Kato T, Yuan ZM.. Role for E2F in DNA damage-induced entry of cells into S phase. Cancer Res 1997; 57: 3640-3643.

39. Müller $\mathrm{H}$, Helin $\mathrm{K}$. The E2F transcription factors: key regulators of cell proliferation. Biochim Biophys Acta 2000; 1470: M1-12.

40. Wu L, Timmers C, Maiti B, Saavedra HI, Sang L, Chong GT et al. The E2F1-3 transcription factors are essential for cellular proliferation. Nature 2001; 414: 457-462.

41. Halaban R, Cheng E, Smicun Y, Germino J. Deregulated E2F transcriptional activity in autonomously growing melanoma cells. J Exp Med 2000; 191: 1005-1016.

42. Nelson MA, Reynolds SH, Rao UN, Goulet AC, Feng Y, Beas A et al. Increased gene copy number of the transcription factor E2F1 in malignant melanoma. Cancer Biol Ther 2006; 5 : 407-412.

43. Alla V, Engelmann D, Niemetz A, Pahnke J, Schmidt A, Kunz M et al. E2F1 in melanoma progression and metastasis. J Natl Cancer Inst 2010; 102: 127-133.

44. Bhatia B, Hsieh M, Kenney AM, Nahlé Z. Mitogenic Sonic hedgehog signaling drives E2F1dependent lipogenesis in progenitor cells and medulloblastoma. Oncogene 2011; 30: 410-422.

45. Bhatia B, Potts CR, Guldal C, Choi S, Korshunov A, Pfister S et al. Hedgehog-mediated regulation of PPAR $\gamma$ controls metabolic patterns in neural precursors and shh-driven medulloblastoma. Acta Neuropathol 2012; 123: 587-600.

46. Regl G, Kasper M, Schnidar H, Eichberger T, Neill GW, Philpott MP et al. Activation of the BCL2 promoter in response to Hedgehog/GLI signal transduction is predominantly mediated by GLI2. Cancer Res 2004; 64: 7724-7731.

47. Kabbarah O, Nogueira C, Feng B, Nazarian RM, Bosenberg M, Wu M et al. Integrative genome comparison of primary and metastatic melanomas. PLoS One 2010; 5: e10770.

48. Johnson DG, Ohtani K, Nevins JR. Autoregulatory control of E2F1 expression in response to positive and negative regulators of cell cycle progression. Genes Dev 1994; 8 : 1514-1525.

49. Kinzler KW, Vogelstein B. The GLI gene encodes a nuclear protein which binds specific sequences in the human genome. Mol Cell Biol 1990; 10: 634-642.

50. Winklmayr M, Schmid C, Laner-Plamberger S, Kaser A, Aberger F, Eichberger $T$ et al. Non-consensus GLI binding sites in Hedgehog target gene regulation. BMC. Mol Biol 2010; 11: 2.

51. Peterson KA, Nishi Y, Ma W, Vedenko A, Shokri L, Zhang X et al. Neural-specific Sox2 input and differential Gli-binding affinity provide context and positional information in Shh-directed neural patterning. Genes Dev 2012; 26: 2802-2816.

52. Chen JK, Taipale J, Young KE, Maiti T, Beachy PA. Small molecule modulation of Smoothened activity. Proc Natl Acad Sci USA 2002; 99: 14071-14076. 
53. Wu J, Kharebava G, Piao C, Stoica BA, Dinizo M, Sabirzhanov B et al. Inhibition of E2F1/CDK1 pathway attenuates neuronal apoptosis in vitro and confers neuroprotection after spinal cord injury in vivo. PLoS One 2012; 7: e42129.

54. Konishi Y, Bonni A. The E2F-Cdc2 cell-cycle pathway specifically mediates activity deprivation-induced apoptosis of postmitotic neurons. J Neurosci 2003; 23: 1649-1658.

55. Eichberger T, Sander V, Schnidar H, Regl G, Kasper M, Schmid C et al. Overlapping and distinct transcriptional regulator properties of the GLI1 and GLI2 oncogenes. Genomics 2006; 87: 616-632.

56. Locker M, Agathocleous M, Amato MA, Parain K, Harris WA, Perron M. Hedgehog signaling and the retina: insights into the mechanisms controlling the proliferative properties of neural precursors. Genes Dev 2006; 20: 3036-3048.

57. Emanuel S, Rugg CA, Gruninger RH, Lin R, Fuentes-Pesquera A, Connolly PJ et al. The in vitro and in vivo effects of JNJ-7706621: a dual inhibitor of cyclin-dependent kinases and aurora kinases. Cancer Res 2005; 65: 9038-9046.

58. Pandolfi S, Montagnani V, Penachioni JY, Vinci MC, Olivito B, Borgognoni L et al. WIP1 phosphatase modulates the Hedgehog signaling by enhancing GLI1 function. Oncogene 2013; 32: 4737-4747.

59. Santini R, Pietrobono S, Pandolfi S, Montagnani V, D'Amico M, Penachioni JY et al. SOX2 regulates self-renewal and tumorigenicity of human melanoma-initiating cells. Oncogene 2014; 33: 4697-4708.

60. Jochemsen AG. Reactivation of p53 as therapeutic intervention for malignant melanoma Curr Opin Oncol 2014; 26: 114-119.

61. Lu M, Miller $P$, Lu X. Restoring the tumour suppressive function of $p 53$ as a parallel strategy in melanoma therapy. FEBS Lett 2014; 588: 2616-2621.
62. Qiu S, Cai Y, Gao X, Gu SZ, Liu ZJ. A small peptide derived from p53 linker region can resume the apoptotic activity of p53 by sequestering iASPP with p53. Cancer Lett 2015; 356: 910-917.

63. Melillo RM, Helin K, Lowy DR, Schiller JT. Positive and negative regulation of cell proliferation by E2F-1: influence of protein level and human papillomavirus oncoproteins. Mol Cell Biol 1994; 14: 8241-8249.

64. Agren M, Kogerman P, Kleman MI, Wessling M, Toftgård R. Expression of the PTCH1 tumor suppressor gene is regulated by alternative promoters and a single functional Gli-binding site. Gene 2004; 330: 101-114.

65. Crooks GE, Hon G, Chandonia JM, Brenner SE. WebLogo: a sequence logo generator. Genome Res 2004; 14: 1188-1190.

(c) (i) (2)(2) This work is licensed under a Creative Commons Attribution-NonCommercial-ShareAlike 4.0 International License. The images or other third party material in this article are included in the article's Creative Commons license, unless indicated otherwise in the credit line; if the material is not included under the Creative Commons license, users will need to obtain permission from the license holder to reproduce the material. To view a copy of this license, visit http://creativecommons.org/licenses/by-nc-sa/4.0/

Supplementary Information accompanies this paper on Cell Death and Differentiation website (http://www.nature.com/cdd) 